\title{
Personality Psychology: Lexical Approaches, Assessment Methods, and Trait Concepts Reveal Only Half of the Story-Why it is Time for a Paradigm Shift
}

\author{
Jana Uher
}

Published online: 8 February 2013

(C) The Author(s) 2013. This article is published with open access at Springerlink.com

\begin{abstract}
This article develops a comprehensive philosophy-of-science for personality psychology that goes far beyond the scope of the lexical approaches, assessment methods, and trait concepts that currently prevail. One of the field's most important guiding scientific assumptions, the lexical hypothesis, is analysed from metatheoretical viewpoints to reveal that it explicitly describes two sets of phenomena that must be clearly differentiated: 1) lexical repertoires and the representations that they encode and 2) the kinds of phenomena that are represented. Thus far, personality psychologists largely explored only the former, but have seriously neglected studying the latter. Meta-theoretical analyses of these different kinds of phenomena and their distinct natures, commonalities, differences, and interrelations reveal that personality psychology's focus on lexical approaches, assessment methods, and trait concepts entails a) erroneous meta-theoretical assumptions about what the phenomena being studied actually are, and thus how they can be analysed and interpreted, b) that contemporary personality psychology is largely based on everyday psychological knowledge, and $c$ ) a fundamental circularity in the scientific explanations used in trait psychology. These findings seriously challenge the widespread assumptions about the causal and universal status of the phenomena described by prominent personality models. The current state of knowledge about the lexical hypothesis is reviewed, and implications for personality psychology are discussed. Ten desiderata for future research are outlined to overcome the current paradigmatic fixations that are substantially hampering intellectual innovation and progress in the field.
\end{abstract}

Keywords Personality $\cdot$ Meta-theory $\cdot$ Methodology $\cdot$ Lexical hypothesis $\cdot$ Lexical approach $\cdot$ Traits $\cdot$ Personality assessment $\cdot$ Philosophy of science $\cdot$ Big five model $\cdot$ Five factor model

\footnotetext{
J. Uher $(\square)$

Comparative Differential and Personality Psychology, Freie Universität Berlin, Habelschwerdter Allee 45, 14195 Berlin, Germany

e-mail: mail@janauher.com
} 
Science provides a special way of constructing knowledge about the world. Unlike nonscientific knowledge construction, science provides a way of thinking simultaneously about phenomena and the means of producing knowledge about them - this presupposes meta-theory and methodology (Althusser and Balibar 1970; Toomela 2011). Meta-theory refers to the philosophical assumptions about the theoretical nature of the phenomena to be studied and to the questions that are asked about them. Methodology refers to the ways (i.e., approaches) in which these questions can be answered and to the techniques (i.e., methods) that can therefore be used (Sprung and Sprung 1984).

All sciences have meta-theories. They determine which elements of real phenomena can be reduced to precisely those subsets of elements that are considered relevant to and defining of concrete scientific phenomena and the ways in which they can be reduced (Althusser and Balibar 1970, p. 84, analysing the philosophy of science of Marx 1867; Køppe 2012; Weber 1949). Metatheories determine what is considered data in a particular field (in a particular historical time; Kuhn 1962), and how the thus-defined data can be analysed and interpreted (Køppe 2012; Wagoner 2009). Hence, the first step of reducing real elements into facts is already a theoretical decision (Weber 1949, p. 173). "Alles Faktische ist schon Theorie"-all facts are already theory (Goethe 1907, p. 127). In other words, "it is the theory which decides what can be observed" (Einstein to Heisenberg in 1926, cited in Heisenberg 1989, p. 10). Meta-theories and methodologies are the rules that govern the effective practices of sciences. Therefore, they should be explicated to enable researchers to constantly scrutinise all levels from epistemology and ontology up to the specific theories about the phenomena studied (Toomela 2011). This also includes rethinking the very reduction of real phenomena into scientific phenomena (Utz 2005).

Contemporary psychology largely follows meta-theory and methodology implicitly, sometimes even "blindly" (Toomela 2011, p. 22) - this occurs in particular when research methods are decided first and research questions are adapted to the methods rather than vice versa (Omi 2012; Westen 1996). "The understanding that research methodology comprises an essential part of scientific theories about phenomena that are studied is not always brought into the center of theoretical reasoning" (Toomela 2009, p. 45). Ignoring the meta-theoretical assumptions underlying particular methods can result in mismatches between methods and research questions that hamper the scientific understanding and the explanations of the phenomena being studied-and thus scientific progress (Loftus 1996; Toomela and Valsiner 2010; Weber 1949). More profoundly, a priori decisions on methods preclude posing the primary question of what the phenomena to be studied actually are (Toomela 2011).

These meta-theoretical and methodological challenges are characteristic of contemporary personality psychology and of taxonomic personality research in particular (Uher 2008a, b, 2011a, b; Uher, Methodological approaches to personality taxonomies: The Behavioural Repertoire x Environmental Situations Approach-A nonlexical alternative, unpublished). The "discovery" of five major dimensions of individual differences is considered a milestone in modern Western psychology (De Raad 1998; Digman 1990; Goldberg 1990). This "break through" was made possible by the assumption that people encode in their everyday languages all those individual 
differences that they perceive as most salient in everyday encounters and that they consider to be socially relevant. This so-called lexical hypothesis, first articulated by Galton (1884), has provided a stringent rationale for using the lexica of human languages as finite sources of information to unravel a few major dimensions of individual differences (Allport and Odbert 1936; Cattell 1943). In English and some other languages, the reduction of the pertinent lexical repertoires to five major dimensions has received the most support in Western scientific communities (Goldberg 1993; John et al. 1988). The lexical hypothesis also suggests that enquiring about the everyday psychological ideas that people develop of themselves and of other individuals could be a suitable method for the scientific measurement of personality (Block 2010; Westen 1996). Assessments by laypeople have become the standard methods of investigation (Baumeister et al. 2007; Matthews et al. 2003) and the "primary source of data" in personality psychology (Schwarz 1999, p. 93). The assumptions expressed in the lexical hypothesis might also contribute to the fact that the prevailing strategies of the scientific explanation of individual differences largely follow structures that are deeply rooted in everyday psychology.

In spite of its enormous importance as one of the most widely used theoretical assumptions to have guided personality psychology (Ashton and Lee 2005), the lexical hypothesis has remained untested (Toomela 2010a; Westen 1996), and still today, its statements have been considered only partially. This article systematically explores the meta-theoretical assumptions that underlie this hypothesis. It starts by highlighting the explicit reference of the lexical hypothesis to two sets of phenomena that must be clearly differentiated; these are, on the one hand, people's lexical repertoires and the representations that these repertoires encode, and, on the other hand, the kinds of phenomena that are perceivable in everyday life and that are being represented. Thus far, personality psychology has focused primarily on just one of these two sets - on lexically encoded representations - but has failed to systematically investigate the second set, namely, the kinds of phenomena that are being perceived and represented.

This article elaborates the distinct natures of the different kinds of phenomena to which the lexical hypothesis refers and explores their commonalities, differences, and interrelations from a philosophy-of-science perspective. This allows for scrutiny to be applied to the phenomena that are being lexically encoded and sheds new light on the fundamental questions of which kinds of phenomena can actually be captured by assessments - and which ones cannot. These analyses reveal that erroneous metatheoretical assumptions underlie the established beliefs about what these phenomena actually are. They show that contemporary personality psychology is largely based on everyday psychological knowledge. Furthermore, the present meta-theoretical analyses allow us to scrutinise the prevailing psychological strategy of explaining individual differences - explicitly or implicitly_by assuming the existence of "traits". The philosophy-of-science perspective identifies explanations based on trait concepts as fundamentally circular. These findings seriously challenge the established assumptions about the causal and universal status of the phenomena described by prominent personality models.

Various researchers have carefully crafted serious concerns about the established five factor models of personality (e.g., Block 1995, 2001, 2010; Eysenck 1992; McAdams 1992; Westen 1996), about assessment data and their interpretation (e.g., Brower 1949; 
Michell 1997, 2003; Omi 2012; Rosenbaum and Valsiner 2011; Schwarz 2009; Trendler 2009; Valsiner 2012), and about the explanations provided by trait psychology (e.g., Allport 1961; Bock 2000; Cervone et al. 2001; Lamiell 2003; Mischel and Shoda 1994, 1995). This article moves these critical objections coherently to meta-theoretical and methodological levels of consideration. It expands them substantially from the philosophy-of-science perspective and elaborates a fundamental critique of the lexical approaches, assessments by laypeople, and trait concepts that currently dominate personality psychology. It closes by outlining 10 desiderata for future research to overcome the limitations revealed in the analyses and to stimulate new directions in the field.

\section{Lexical Encodings-Constructs and Representations}

The lexical hypothesis states that people encode in their everyday languages all those differences between individuals that they perceive to be salient and that they consider to be socially relevant in their everyday lives. Encodings about differences within individuals over time are not explicitly mentioned, however (see below). Among the many phenomena that individuals can perceive, recurrent patterns are particularly meaningful because such patterns may allow predictions of future events while facing the uncertainty of the future. Individuals therefore seek to identify recurrent patterns in their experiences with their personal world (Kelly 1955). To describe, explain, and to predict the likely occurrences of events, people construct and represent their experiences in private ideas (Valsiner 2012). These ideas are called subjective or individual representations (Jovchelovitch 2007; Moscovici 1984). Representations referring to experiences with one's own person and the - especially social—world are called personal constructs in personality psychology (Kelly 1955).

To communicate their perceptions and mental constructs of what they perceive and to negotiate socially shared meanings in order to cope with the world collectively, groups of individuals create and use social constructs. These constructs are called intersubjective or social representations (Jovchelovitch 2007) or folk concepts in personality psychology (Tellegen 1993). Constructs and representations referring to the perceptions of persons are called personality constructs. The lexical hypothesis states that, over time, socially shared constructs of self- and other-perception become encoded in the natural human languages. Pertinent lexical encodings reflect the body of everyday psychological ideas, beliefs, values, and practices that people have developed about individuals (Block 2010; Wagner et al. 1999; Westen 1996).

\section{What is Constructed and Represented-Phenomena and Patterns}

What is it that is constructed and represented as "personality"? Psychological definitions of personality - a few prominent ones picked out of many-provide the first insights into what scientific psychologists construct as personality. Some frequently cited definitions refer fairly generally and descriptively to personality as

those characteristics that account for a person's consistent patterns of feeling, thinking, and behaving (Pervin and John 1997, p. 4) or as 
an individual's characteristic patterns of thought, emotion, and behaviour, together with the psychological mechanisms-hidden or not-behind those patterns (Funder 2004, p. 5).

These definitions include psychological and behavioural phenomena. Allport (1937) also included psychophysical systems and environmental adaptivity by defining personality as

the dynamic organization within the individual of those psychophysical systems that determine his unique adjustments to his environment (p. 48).

Eysenck (1947) furthermore specified genetic and environmental causality, biological systems, and ontogenetic development when he defined personality as

the sum-total of the actual or potential behaviour-patterns of the organism, as determined by heredity and environment; [that] originates and develops through the functional interaction of the four main sectors into which these behaviourpatterns are organized: the cognitive sector (intelligence), the conative sector (character), the affective sector (temperament), and the somatic sector (constitution) (p. 25).

McCrae and Costa (2008) extended the scope of consideration by conceptualising particular personality constructs as reflecting "universals" of human nature that are "invariant across human cultures" (McCrae and Costa 1997, p. 510), and thus also potentially phylogenetic in origin (McCrae 2009).

These definitions are sufficient for highlighting fundamental issues. First, they refer descriptively to particular patterns (e.g., those described as "characteristic" and "consistent") and to the different kinds of phenomena in which these patterns occur (e.g., physiological, psychological, and behavioural phenomena). Second, they include assumptions about the causation of the described patterns in the described phenomena by other kinds of phenomena internal to the individual (e.g., psychological phenomena) and/or external (e.g., environmental conditions) considering various explanatory perspectives (e.g., proximate, adaptive, ontogenetic, phylogenetic). Astonishingly, there is no mention of people's lexical encodations and representations of individual differences although these are central to the lexical hypothesis.

From philosophy-of-science perspectives, the different kinds of phenomena studied with regard to personality must be clearly differentiated. This is essential because interrelations among them can be untangled only if they are explored each in their own right and if a priori assumptions about specific interrelations are avoided. This is rarely done in psychology. Behaviourists have focused too much on external conditions and behaviours, but have largely ignored psychological phenomena. Subsequent researchers have tried to overcome the limitations of behaviourism and have therefore focused strongly on psychological phenomena and on individuals' representations of their world, but behaviour has faded into the background. The prevailing investigative strategies are preoccupied with causal processes and the conditions in which behavioural phenomena 
occur-especially psychological ones-with the result that clear differentiations of psychological and behavioural phenomena are missing.

The meta-theoretical definition of behavioural phenomena as "external activities or externalisations of living organisms that are functionally mediated by the environment (Millikan 1993) in the present" (Uher, What is behaviour? And (when) is language behaviour? A meta-theoretical definition, unpublished) allows such differentiations. This definition highlights the intrinsic relatedness of behaviour to properties of the immediate external environment that are defined as environmental situations and emphasises that it is inherently bound to the present. Externality differentiates behaviour from thoughts, emotions, and other psychological phenomena, which are also bound to the present (Gillespie and Zittaun 2010; Toomela 2010a; Valsiner 1998, 2012) but are internal to the individual (Toomela 2008). This metatheoretical definition generally defines all behavioural phenomena without specifying particular ones (e.g., goal-directed action or response) because many of these concepts include a priori assumptions about causally related internal processes (e.g., psychological phenomena) and external conditions in the environment (e.g., particular stimuli) that are separate kinds of phenomena. To scrutinise which kinds of phenomena people can perceive in everyday life and construct as personality, it is helpful to first specify the particular patterns referred to in definitions of personality.

\section{Defining Patterns}

For scientific definitions, the notions of "a person's consistent patterns", "individual characteristics", and "individual uniqueness" are surprisingly vague. They do not specify what is meant to be "consistent" with what, nor do they indicate which patterns are considered "different, "unique", and "characteristic" and why. Moreover, they fail to mention within-individual variability and structural complexity - both within and between individuals. This is remarkable because these patterns refer to phenomena (e.g., those of the psyche and of behaviour) that are heterogeneous, complex, dynamic, and thus highly fluctuating. In such phenomena, differences among individuals are necessarily apparent at any given time (Uher 2011a) and determinations of meaningful consistency - both within and between individuals - are matters of mere convention. Meta-theoretical analyses help to carve out basic criteria for determining which patterns can be considered specific to particular individuals, and thus different from others, which ones cannot, and why. So what does individual-specificity mean?

Probabilistic Patterns In the steadily fluctuating flow of events in highly dynamic phenomena, individuals necessarily show considerable within-individual variability over time. Consequently, they can be characterised only by probabilistic patterns. What types of probability ${ }^{1}$ are able to describe these patterns? First, events of psychological and behavioural phenomena are not random, such as events in throws of dice. There are no equipossible elementary events in well-defined sample spaces that can be studied in repeated experiments as in Laplacian theory. Moreover, the

\footnotetext{
${ }^{1}$ For the present meta-theoretical analyses, theories considering subjectivistic probabilities of the occurrences of events (e.g., degrees of belief; Gillies 2000; Rychlak 1968) will not be considered here (for reasons described in detail below).
} 
events are not independent from one another - this applies to events of the same phenomenon (e.g., several smiles) as well as to events of different phenomena (e.g., a smile and a laugh). Rather, different events may co-occur and even depend upon one another, particularly if the phenomena are functionally similar or related. Their events need not be exclusively disjunctive (e.g., smiles and laughs). Thus, finite (limiting) frequencies, which are defined as empirical occurrences relative to all possible occurrences (von Mises 1928), are inadequate for describing individual probabilities in psychological and behavioural phenomena.

The concept of propensity probabilities seems to be better suited for this purpose. Propensities are considered properties of physical objects or situations that are assumed to cause empirical frequencies, even if only single-case observations are made (Popper 1959). They are therefore conceived also as properties of repeatable conditions that determine empirical frequencies in the long run (Gillies 2000). ${ }^{2}$ But this very assumption precludes the idea that propensity probabilities can be applied to individuals because their defining property as living systems is the fact that they undergo continuous changes during ontogenetic development. This is true for the molecules in their bodies and the phenomena constructed as personality alike. In fact, personality is assumed to develop and change gradually over the course of the lifespan (e.g., Caspi and Roberts 2001; Cattell 1950; Soldz and Vaillant 1999). This implies that the individual's empirical frequencies cannot converge (i.e., stabilise) in the long run as may be true for physical systems. By contrast, the probabilities themselves change over time - an idea typically not considered by theories of propensity probabilities (cf. Gillies 2000; Popper 1959).

Further differences are essential. In psychological and behavioural phenomena, there are no discrete events such as there are in coin tosses. Well-defined natural entities on which probability estimations could be based are lacking. Instead, it is the scientists who must decide which entities of real phenomena make up particular scientific phenomena. The ways in which they define entities as categories depend not only on the phenomena themselves, but also on the purposes of their reduction (Weber 1949). Moreover, the processual character and microgenetic development of many psychological and behavioural phenomena (Rosenthal 2004) entails that their occurrences can be construed as frequencies only in some cases. In other cases, they may be construed more accurately as durations of nonfixed and often highly variable length (or as latencies, which are durations with specified start times).

Consequently, in fluctuating phenomena such as those of the psyche and of behaviour, events can be considered only by their empirical occurrences (i.e., in terms of frequencies and durations) relative to particular time periods. This new probability type will therefore be called a time-relative probability. For example, in observations of free play in a kindergarten group, a time-relative probability of $10 \mathrm{~min}$ per hour of observation time during which a child plays by him/herself may be determined for a particular 5-year-old child. The time periods in which timerelative probabilities are determined must be specified precisely-both in terms of the occasions and spans of time in which the data were collected to determine the probabilities and in terms of historical times in the individual's ontogeny. Both are

\footnotetext{
${ }^{2}$ The concept of propensity probabilities reifies observed properties into causal entities of an unkown and undefined kind. As such, the explanatory strategy is inherently circular and faces the same challenges as those elaborated for trait psychology in this article.
} 
essential to define individual-specificity. For example, the 10 min per hour probability could have been determined from $20 \mathrm{~h}$ of regular observation obtained across a time period of 2 weeks; the ontogenetic stage of the individual is specified by an age of 5 years, which can be categorised as early childhood.

This new probability type enables ratio-scaled quantifications that are essential for quantitative comparisons across time and situational contexts and between individuals, groups, populations, and species (Uher, Meta-theoretical foundations of objectivity versus subjectivity in quantifications of behaviour and personality; An integrative meta-theoretical framework for research on individual behaviour in context - situations, populations, species, both unpublished). It also allows for the merging of concepts of averages across occasions, as studied in personality research, with concepts of ranges, especially maxima, as studied in intelligence and achievement research ${ }^{3}$ (Ackerman 1994), and concepts of variability in terms of differences between consecutive occasions (i.e., fluctuations) as studied in physiological and behavioural research (De Weerth et al. 1999). For example, the child's time-relative probability could be on average $10 \mathrm{~min}$ per hour, ranging from 5 to $20 \mathrm{~min}$ per hour on a daily basis; the magnitude of day-to-day variability (for comparable occasions across the days) as indicated by the coefficient of variation could be $C V=0.5$. This coefficient specifies the standard deviation standardised by the mean (because the standard deviation is sensitive to the sample mean) to allow for comparisons between different samples (for an overview of methods of analyses of variability, see van Geert and van Dijk 2002). Such comprehensive analyses of patterns are important because within-individual variability in psychological and behavioural phenomena is pronounced and bears theoretical and empirical importance. For example, within-individual variability can indicate ongoing processes and can reflect phenomena that are important for explaining changes and development (van Geert and van Dijk 2002, p. 344). Moreover, within-individual variability often substantially exceeds between-individual variability (Shweder and Sullivan 1990; Uher, An integrative meta-theoretical framework for research on individual behaviour in context—situations, populations, species, unpublished).

Differential Patterns Time-relative probabilities that characterise all individuals in the same way cannot be individual-specific. If all children in the observed group have the same time-relative probability of self-playing, then these probabilities cannot characterise any one of them individually. Time-relative probabilities can reflect individual uniqueness only if they deviate from those of other individuals of a particular reference group of interest (e.g., a social group, culture, or species) - that is, if they are differential. This is the case if the determined time-relative probabilities differ between children such that their individual averages vary interindividually, for example, between $2 \mathrm{~min}$ and $30 \mathrm{~min}$ per hour, their individual ranges vary, for example, between $15 \mathrm{~min}$ and $50 \mathrm{~min}$, and their individual within-individual variabilities vary, for example, between $C V=0.3$ and 1.3.

The concept of time-relative probabilities presupposes that the number of occasions and periods of time considered for the probability estimations are comparable

\footnotetext{
${ }^{3}$ Personality research is often distinguished from intelligence research in American psychology. In European traditions (e.g., Cattell, Eysenck, Stern, Pawlik), intelligence and achievement are considered inherent parts of an individual's personality.
} 
between individuals. This is important when considering the effects of aggregation on the reliability of probability estimations (cf. Spearman 1910) and when taking into account the fact that the individuals' probabilities themselves may gradually change over time (i.e., develop). In the given example, this means that the time-relative probabilities of the other children should also be estimated based on $20 \mathrm{~h}$ of observation across a time span of 2 weeks. These children should also be of similar age in order to disentangle differences between individuals from differences between age groups. Furthermore, the situational contexts in which different individuals are studied should be comparable between them because the average time-relative probabilities of particular groups of individuals may generally shift across different situations (Uher, An integrative meta-theoretical framework for research on individual behaviour in context-situations, populations, species, unpublished.) Analyses of differentiality therefore require statistical standardisation of time-relative probabilities within the time periods and within the situational contexts in which they are determined (Uher 2011a). Statistical standardisation removes the information on absolute time-relative probability scores, however, because the data are converted into relative, but still ratio-scaled data. Yet, the absolute scores can always be traced when needed for interpretation and for later comparisons with other samples or with future investigations of the same sample.

Temporal Patterns Differential patterns in time-relative probabilities that change rapidly cannot characterise an individual's uniqueness. To reflect individualspecificity, they must be stable across time periods that are longer than those in which the probabilities were first ascertained. As with the analyses of differential patterns, the analyses of temporal patterns presuppose that probability estimations are based on numbers of occasions and periods of time that are comparable between the individuals being studied and between the time periods being contrasted. In the above example, this means that the differential patterns in the children's time-relative probabilities for self-play should be similar when estimated again some time later in a second period of 2 weeks. Temporal stability is an essential prerequisite for justifying interpretations of the obtained standardised aggregate scores as reflecting individual-specific patterns (Uher 2011a).

Temporal stability can be analysed for differential patterns in the individuals' averages, ranges, and variabilities. For example, it is quite possible that individuals with similar differential scores in their average probabilities will differ from one another in stable ways with regard to their ranges and patterns of within-individual variability. The magnitude of temporal stability considered meaningful to construct individual-specificity is necessarily a matter of convention that can and must be explicitly defined. It depends on the ontogenetic stages of the individuals under study as well as on their species-specific life expectancy (Uher 2009). It also depends on the phenomena being studied and the meta-theory used. For example, in biology, weak test-retest correlations of individual differences in behaviour across just a few days or weeks of $r=.20$ are considered ecologically and evolutionarily meaningful (Sih and Bell 2008), whereas in human psychology, such weak correlations are considered indicators of unreliable measurements of individual-specific patterns (Uher 2011a).

In conclusion, for fluctuating and dynamic phenomena such as those of the psyche and behaviour, individual-specificity refers to differential patterns in time-relative 
probabilities that are stable in ways that are considered to be meaningful. This concept fits well into Brunswik's (1955) probabilistic theory for functional psychology. Brunswik ascertained that although the environment may be lawful in terms of physical principles, to the individual, "it presents itself as semi-erratic ... therefore all functional psychology is inherently probabilistic" (Brunswik 1955, p. 193). An empirical application of this meta-theoretical concept of individual-specificity to behaviour was demonstrated in a study on capuchin monkeys based on 141 contextualised behavioural variables (Uher et al., Contextualised behavioural measurements of personality differences obtained in behavioural tests and social observations in adult capuchin monkeys (Cebus apella), unpublished).

Individual-specific patterns that have been identified in these ways can be subsequently studied across longer periods of time to explore patterns in their gradual change and ontogenetic development (Uher 2011a). This presupposes again that the occasions and time spans as well as the historical times that are studied should be specified and should be comparable between the individuals under study.

The concept of time-relative probabilities allows for the close examination of individual cases in specified situations. By studying many individuals in this way, it also allows for the study of lawful patterns at the sample level. The former type of study is commonly referred to as ideographic; the latter one as nomothetic. But ideographic approaches in terms of single case studies in and of themselves need not be informative about particular individuals' specificity because they fail to disentangle individual-specific from group-, population-, or species-specific patterns (Uher, An integrative meta-theoretical framework for research on individual behaviour in context — situations, populations, species, unpublished). Single case studies are informative about individual-specificity only if they are based on the meta-theoretical assumption that individuals generally differ from one another in a given population. This is commonly assumed for humans, but not necessarily for individuals of other species. Single case studies of, for example, cockroaches can also be based on the assumption that all individuals are alike. Thus, studying one individual is assumed to provide information about all individuals, but not necessarily about the peculiarities of a particular one. Ideographic approaches are therefore informative about individual-specificity only if they are conceptualised as ideographic-nomothetic approaches such as in the above-introduced concept of individual-specificity that is based on differential patterns in time-relative probabilities that are stable in meaningful ways.

Up to this point in the article, psychological and behavioural phenomena have been discussed only exemplarily to illustrate the meta-theoretical analyses of individual-specificity in fluctuating phenomena. I will now scrutinise the particular kinds of phenomena in which individual-specific patterns are constructed as personality. According to the lexical hypothesis, the lexically encoded representations of individual-specificity refer to phenomena that are (1) perceivable and salient and (2) important in everyday life, in particular in social encounters (John et al. 1988). Systematic explorations of the kinds of phenomena to which these criteria apply encounter two fundamental challenges.

\section{Fundamental Challenges}

With regard to phenomena that people perceive and construct as personality, one must consider that individual-specific patterns cannot be directly perceived at any given 
moment in any given individual because they refer to probabilistic, differential, and temporal patterns. This has far-reaching implications for their investigation in particular in phenomena as dynamic and fluctuating as those of the psyche and of behaviour (discussed in detail below; Michell 1997, 1999; Rosenbaum and Valsiner 2011; Uher, Meta-theoretical foundations of objectivity versus subjectivity in quantifications of behaviour and personality, unpublished).

With regard to people's constructs and representations of these phenomena, one must consider that the construing activity of the human mind leads people to overlook and to transcend gaps in perception (Brunswik 1952; Daston and Galison 2007; Valsiner 2012). These are challenges for scientific psychologist as well. In contrast to scientists in many other disciplines, personality psychologists study phenomena that are important in everyday life - including their own — in individuals of their own kind. They start researching these phenomena and such individuals only after having acquired a substantial everyday psychological knowledge base and a pertinent lexical repertoire in their pre-academic lives (Uher 2011a). Their everyday psychological knowledge and the sociocultural context in which it is embedded may inevitably influence and "confuse" their thinking as researchers (James 1893, p. 196; Weber 1949, p. 54) —despite all scholarly efforts (Jovchelovitch 2007; Komatsu 2012; Valsiner 2012).

These challenges, which will be further explored below, require precise specification and clarification of the central statements of the lexical hypothesis. To identify individual-specificity, (1) people must be able to directly perceive the phenomena in question in individuals. Only then can they consider the occurrences of their events and compare these between individuals to identify differential patterns in timerelative probabilities; and only then can they consider temporal patterns thereinthe three meta-theoretical criteria of individual-specificity in fluctuating and dynamic phenomena (Uher, Meta-theoretical foundations of objectivity versus subjectivity in quantifications of behaviour and personality, unpublished). This is also implied by the assumption that it is salient phenomena that become lexically encoded over timesalience presupposes direct perceivability. The notion of perceiving does not imply intention, purpose, or conscious awareness - in contrast to observing. (2) Directly perceivable phenomena can become socially relevant and important in everyday life only if these phenomena are also directly involved in the individuals' interactions with and in their relations to their - in particular social — environment. If particular phenomena cannot directly interact with an individual's environment but depend completely on mediation through other phenomena, then these former phenomena in and of themselves cannot become socially relevant and important in everyday life without the phenomena that mediate them. Thus, they are secondary. For example, by itself, a private thought cannot become effective in an individual's life unless the individual eventually externalises it in language or acts upon it (Uher, What is behaviour? And (when) is language behaviour? A meta-theoretical definition, unpublished). This issue will be discussed in detail below.

\section{Scrutinising Phenomena}

To which kinds of phenomena do these two criteria-(1) direct perceivability and (2) direct involvement in the individual's environmental relations - apply? The lexical 
hypothesis specifies no particular kinds of phenomena, but the above-mentioned psychological personality definitions do.

Psycho-Physiological Phenomena Some definitions specify psycho-physiological phenomena. Individuals cannot directly perceive any of these phenomena, however, either in themselves or in any other individual (criterion 1 failed). Moreover, psychophysiological phenomena may be involved only indirectly in the individuals' relations to their environment and depend on mediation by other phenomena, such as of physiology (e.g., metabolic) and of behaviour (criterion 2 partly failed; see Fig. 1).

Psychological Phenomena Psychological phenomena, such as those described as emotions, thoughts, motives, beliefs, attitudes, and many others are essential elements of many psychological personality definitions. But all these phenomena are entirely internal (Toomela 2008). They cannot directly interact with any of the individual's external systems (Bronfenbrenner 1979). Hence, by themselves, psychological phenomena cannot be directly involved in the individuals' environmental relations (criterion 2 failed). And because they are entirely internal, people can perceive them

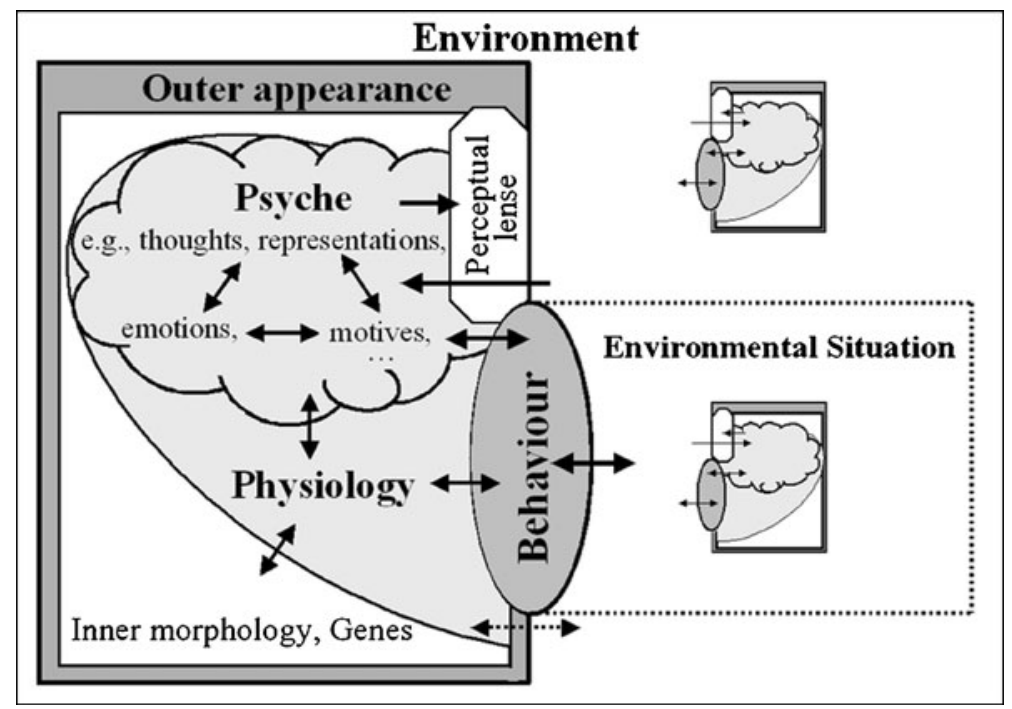

Fig. 1 Behaviour - The essential bridge from the psyche to the environment. Note. The box in the dark frame on the left side of the figure and the two smaller boxes that mirror its shape and composition on the right side symbolise individuals. The arrows indicate the directions of the interactions that are possible between the different types of phenomena considered; in the smaller symbols, only some of them are depicted. Of the kinds of phenomena frequently mentioned in personality definitions, phenomena of behaviour and of outer appearance are the only ones that fulfil both criteria implied by the lexical hypothesis - i.e., (criterion 1) they are directly perceivable by individuals in themselves and in other individuals, and (criterion 2) they can be directly involved in the individuals' interactions with and their relations to the environment. Behaviour is the essential interface between an individual's psychological phenomena, which are entirely internal, and that individual's (abiotic and biotic, especially social) environment. Those environmental details that functionally mediate the individual's externalisations in the present (i.e., the individual's behaviour) are called the environmental situation (indicated with a dotted line). In the graphic, the lower of the two individuals (indicated as small boxes) is part of the environmental situation of the individual on the left side (indicated as large box), whereas the upper one is not 
only in themselves through introspection (Wundt 1904), but they are unable to perceive psychological events in any other individual (Locke 1689; Toomela 2008, 2011; criterion 1 semi-fulfilled). The psychological events of others can be inferred only from events of external — and thus directly perceivable — phenomena that may mediate between their internal and external systems, such as from behavioural phenomena (see Fig. 1), including parts of language (Uher, What is behaviour? And (when) is language behaviour? A meta-theoretical definition, unpublished).

Difficulties arise, however, because these mediating phenomena may not reflect psychological phenomena unequivocally or accurately. Similar behavioural phenomena can be related to different psychological phenomena and different behavioural phenomena to similar psychological phenomena (Cervone et al. 2001; Kagan 1994, 1998; Lewin 1935; Mischel and Shoda 1994). Clear meta-theoretical differentiations between behavioural and psychological phenomena are therefore essential (see above). Moreover, differences in behavioural structures may be but are not necessarily associated with differences in psychological structures (Toomela 2011). Further complicating the matter is the fact that individuals must adapt their mediating behaviours to the (subjectively interpreted; Rotter 1954) realities of the physical and social environment_- "acting is not the same as thinking" (Lahlou 2008, p. 21, translated). This means that individuals can (and must) control the mediators to some extent depending on the given contexts. This substantially complicates the ability to make inferences to underlying psychological events. The reduced ability shown by babies and young children to control behaviour may result in stronger relations between the events of their psychological phenomena and those of their behavioural phenomena. Yet their inability to provide self-reports hinders our ability to "validate" any inferences that we make to their underlying psychological events.

But self-reports cannot be used for straightforward inferences either, not even in adults, given how people acquire their semiotic repertoire for describing psychological phenomena during childhood. Children learn the shared meaning of symbols only because adults infer psychological phenomena from the children's behaviours and because they label these inferences with particular symbols that they had learned in the same way. But because they cannot perceive the children's psychological phenomena directly, they may vary - and also err - in their interpretations. Not everyone is "good at understanding" children; that is, at making inferences to their psychological events with relatively high validity.

The horizon of one's own realm of experiences plays an important role as well. Some individuals experience psychological phenomena that others obviously never experience or do not experience in the same way - as reflected in the very assumption of individual differences. How can individuals understand what these symbols that denote particular psychological phenomena refer to if they have never experienced such phenomena themselves? How can colour-blind people understand the actual visual experience of people who are able to perceive particular wavelengths of light (Ludlow et al. 2004)? Locke (1689) ascertained that individual differences in the physical properties of sensory organs should produce different perceptions and thus different ideas in different individuals. But this could never be detected because nobody can pass into another individuals' body to perceive the perceptions that those organs produce. It seems inevitable that even within semiotic communities - individuals develop slightly different personal connotations for socially shared symbols denoting particular psychological phenomena (cf. 
Westen 1996). Consequently, it can never be ascertained whether particular behaviours or particular symbols of shared meaning refer, in fact, to exactly the same psychological phenomena in different individuals. Their presence and quality in others cannot be detected with absolute certainty because every individual can directly access only his or her own psychological phenomena and cannot access anyone else's (Locke 1689).

Behavioural Phenomena Behavioural phenomena, by contrast, are directly perceivable by individuals both in themselves and in other individuals (criterion 1 fulfilled); however, this perception need not be conscious (see notion of perceiving above). As external activities or externalisations that are functionally mediated by the present environment, behavioural phenomena are defined by being directly involved in the individuals' interactions with and in their relations to their environment (criterion 2 fulfilled).

Consequently, among those kinds of phenomena that are frequently incorporated in contemporary personality definitions, behaviour is the only one that fulfils both criteria implied by the lexical hypothesis. For this reason, personality is also conceptualised as "dimensions of behavioural space" (Cattell 1950, p. 221), "individual differences and profiles in behaviour and behavioural performances" (Pawlik 2006, p. 16, translated), and as individual-specific behavioural phenotypes (Uher 2011a).

Phenomena of Outer Appearance Other phenomena of ecto-phenotype fulfil these criteria. Individuals can directly perceive individual-specific patterns in natural outer appearance, such as body size and build, physiognomy, hair, or smell both in themselves and in others (criterion 1 fulfilled). These phenomena can also be directly involved in the individuals' relations to their-in particular social-environment (criterion 2 fulfilled). Artificial modifications that individuals often add to their natural outer appearance to stress their individuality and that are socioculturally considered "adornments" such as clothing, colour, hair style, jewellery, and fragrances, are also - and actually purposefully made to be-(1) directly perceivable by other individuals. They can also be (2) directly involved in the individuals' environmental interactions, their social interactions in particular, for example, as means of social communication ("fine feathers make fine birds") or by influencing how individuals are socially perceived in terms of their "social stimulus value" (Allport and Odbert 1936; Cattell 1950). Hence, ecto-phenotypical phenomena other than behaviour can also become socially relevant in everyday life.

In summary, the meta-theoretical assumptions of the hypothesis that humans have lexically encoded all individual differences in phenomena that are (1) directly perceivable and (2) socially relevant and important in everyday life, and thus directly involved in their environmental interactions, apply only to phenomena of behaviour and outer appearance. But they do not apply to physiological and psychological phenomena.

This highlights two central points in which contemporary personality psychology deviates from the meta-theoretical assumptions of the lexical hypothesis. First, outer appearance is commonly not considered in scientific personality concepts. This is surprising because - as to be expected from the lexical hypothesis - such individual differences are encoded in everyday language. When Allport and Odbert (1936) compiled lexical personality descriptors from Webster's New International Dictionary of the English Language (1925) for first comprehensive lexical studies, 
they also found terms describing physical appearance. Norman (1967) counted 882 such terms in a later edition of that dictionary. According to the lexical hypothesis, "these characteristics should be included in an exhaustive specification of personality" (John et al. 1988, p. 186), but descriptors of outer appearance are typically excluded from lexical personality research (Saucier and Goldberg 1998).

Interestingly, some early personality theorists explicitly considered outer appearance in their definitions of personality. Guilford (1959, p. 7) conceived morphology as one of seven "modalities of traits representing different aspects of personality". Eysenck (1947, p. 25) conceived constitution (the somatic sector) as one of four main sectors of personality. Outer appearance is also central to constitutional theories of personality (regardless of their methodological insufficiencies), such as those focusing on body build (Kretschmer 1977; Sheldon and Stevens 1942), skull shape (phrenology; Bouts 1931), and physiognomy (physiognomics; Aristotle, 300 BC, 1963; Lavater 1775-1778). Outer appearance is also increasingly considered in research on person-perception at zero-acquaintance (Borkenau and Liebler 1992; Hartung and Renner 2011), yet in relation to and not as part of personality.

A second point in which contemporary psychology deviates from the metatheoretical assumptions of the lexical hypothesis is that psycho-physiological and psychological phenomena are frequently incorporated in personality concepts although they do not fulfil the two criteria. Psycho-physiological phenomena (cf. Allport 1937) are not perceivable by individuals in themselves or in others nor are they well represented in the everyday lexica. Psychological phenomena are prominently conceived as (the) core elements of personality both in scientific psychology (Matthews et al. 2003) and in everyday psychology. In fact, the human lexica contain many terms that are (also) used to describe individual-specific patterns in psychological phenomena (e.g., anxious, playful). Granted the lexical hypothesis is valid, so how can this be?

\section{Genesis of Personal Constructs of Self- and Other-Perception}

According to the lens model of human perception (Brunswik 1952, 1955), in a given moment, individuals can perceive from their complex environment only details although they have flexibility regarding which particular details they focus on. Brunswik (1952, p. 23) therefore considered the human perceptual systems to be "imperfect machines" that allow for the creation of only imperfect mental representations. Representational accuracy depends on the ecological validity with which individuals can perceive their environment by shifting the narrow lens filters of their perceptual systems. The accuracy of representations of highly dynamic and fluctuating phenomena, such as behaviour, may be particularly impaired.

However, developing perceptual constructs involves more than merely representing sketchy inputs as modelled in artificial neuronal networks. These networks passively map any input they are receptive to. But perceptual constructs are not imprints that external phenomena breaking through the individuals' perceptual lenses leave on their inner mental images as light breaking through an optical lens darkens photobase paper. Representations are not copies (Jovchelovitch 2007). Mental representations shape and sharpen the individuals' perceptual lens filters, thus changing 
their perception (Fig. 1). Moreover, individuals can also perceive actively. They can focus their perceptual lenses purposefully on particular details that could be meaningful and that they therefore seek to perceive (Brunswik 1952). Particularly meaningful in complex environments are recurrent patterns because their recognition and mental representation may provide orientation and predictive control of future events (Kelly 1955). This may lead individuals to selectively perceive and actively construct more patterns than there actually are in the perceivable phenomena. Similarly, they may seek to primarily perceive those structures and phenomena that match the mental representations that they have already established (e.g., their category systems); whereas they may give less consideration to those that do not match (i.e., thus perceiving "through coloured glasses"). These processes of perception and mental representation are iterative and self-reinforcing.

Essential for individuals are perceptions and constructs of patterns in their experiences with their own person and their own relations to the environment (Kelly 1955). Because individuals can directly perceive psychological phenomena in themselves, they may recognise relations between patterns of their own psychological experiences, patterns of their own behaviours, and patterns of their environment. It may be for this reason that such relations are essential parts of personal constructs. However, phenomena of both the psyche and behaviour are fluctuating and highly dynamic as are many phenomena in the environment, the social environment in particular (Brunswik 1955). The details that individuals can perceive from these phenomena through shifting their perceptual lenses are necessarily inconsistent and fragmentary (Brunswik 1952). Construing coherent and meaningful patterns in these phenomena and in their interrelations must transcend these perceptual gaps.

\section{The Mind-Environment Connection and the Essential Role of Behaviour}

The most fundamental gap is that between the phenomena of the psyche and those of the environment. This gap is particularly puzzling and difficult to understand because it occurs in just one direction. Environmental phenomena may directly influence psychological phenomena through perception. But there is no direct connection in the other direction (Fig. 1). As entirely internal phenomena, psychological phenomena may be able to directly influence other internal phenomena. For example, they may influence physiological phenomena such as in psycho-neuro-endocrinological systems, but they cannot directly influence any phenomenon in the individual's environmental systems (Bronfenbrenner 1979). No thought and no motivation can move an object in physical space. No emotion can directly influence another individual. This is only possible for phenomena that are externalised or external-such as the ectophenotypical phenomena of behaviour (including some parts of language; Uher, What is behaviour? And (when) is language behaviour? A meta-theoretical definition, unpublished) and of outer appearance. Thoughts, motives, emotions, abilities, and all other psychological phenomena can become effective in an individual's environment and in that individual's environmental relations only if these phenomena can somehow reach the external world through mediation of other phenomena at least at some point.

Behavioural phenomena are particularly suited for this mediation because their dynamics and flexibility may be somewhat comparable to those of the psyche. This enables timely mediation to the external world. This nearness-in-time is particularly 
important for the individual's adaptation to and his or her interaction with dynamic and flexibly changing environments, especially in the social domain. Phenomena of outer appearance are comparably less flexible and more static, especially natural outer appearance. Artificial modifications may be more flexible - but they are ultimately results of behaviour. Psychological phenomena cannot directly influence outer appearance; even laughter and worry lines in the face are results of behaviour.

Consequently, behaviour is the essential interface between the individual's internal (biological and psychological) and external (abiotic and biotic, in particular social) systems. It serves as the vital-and for psychological phenomena, the only-bridge between the individual's internal and external worlds (Fig. 1).

No individuals demonstrate the essential nature of this vital bridge and the onesidedness of the mind-environment connection more dramatically than those suffering from locked-in syndrome. Individuals in this condition are patients who are awake and conscious, but who have no means of producing speech, limb, or facial movements because their voluntary control over muscles is (sometimes completely) paralysed (Laureys et al. 2005). This loss of motor control burns the sole bridge between their psyche and the external world. They can still perceive their environment with (almost) all of their senses and continue to construct it in their minds (Laureys et al. 2005). But they cannot behave and thus transmit information from their internal worlds to the external world anymore. Experiencing the mindenvironment connection in its full-blown one-sidedness may perhaps cause their greatest suffering. Locked-in syndrome can be recognised and differentiated from vegetative states only in those patients who are still able to perform minimal behaviours, be it sniffs or eye blinks. Such behaviours, as minuscule as they may be, are mediating channels that enable patients to externalise information from their inner psychological world. This mediation can be successful, however, only if the social environment interprets these externalisations correctly - and if it still tries at all to infer psychological phenomena in seemingly nonbehaving individuals.

The one-sidedness of this connection seems so puzzling and incompatible with the logic of the human mind and, at the same time, behaviour fulfils its mediating function so promptly and so smoothly, that individuals fill this gap (often not knowingly) with assumptions of direct mutual connectedness. When individuals recognise recurrent patterns in their own psyche and in their environment, they frequently construct direct mutual connections and causal associations - seldomly becoming aware of the impossibility of such. Personal constructs of self-perception therefore regularly contain assumptions about direct mutual connectedness and causal relations. These constructs constitute oversimplified representations that are parsimonious and often sufficiently viable in everyday life and that can provide some orientation and predictability in complex and uncertain environments (Kelly 1955). The iterative and self-reinforcing processes of perception and of mental construction contribute to the maintenance of these erroneous assumptions and make the one-sidedness of the mind-environment connection for individuals increasingly harder to recognise.

\section{Preconditions for Identifying Individual-Specific Patterns}

Predictability is particularly important in the social environment. Therefore, individuals seek to perceive and construct patterns that recur in many individuals - that is in 
the average individual — of their social environment. Of particular importance are the environmental interactions of the average individual - that is normative patterns of behaviour and outer appearance. Individuals also seek to recognise patterns that recur in particular individuals and their environmental interactions - that is in individualspecific patterns of behaviour and outer appearance. They construct these individualspecific patterns of environmental interactions as personality (see above; cf. lexical hypothesis; Allport 1937; Gray 1999, p. 563).

But how can individual-specific patterns be constructed at all? In fluctuating phenomena, individual-specificity refers to differential patterns in time-relative probabilities that are meaningfully stable over time (see above). As such, they cannot be perceived at any given moment. "There is no present for all the elements and structures of conceptual systems at once" (Althusser and Balibar 1970, p. 318). Whereas behaviour is defined by its functional reference to the present (Uher, What is behaviour? And (when) is language behaviour? A meta-theoretical definition, unpublished), constructs of personality - because they refer to time-relative probabilistic patterns that are stable across time-also involve phenomena of the past that have already ceased to be. Therefore, the only way in which individuals can capture personality is to - literally - (re)construct someone's personality based on individualspecific patterns that they can (re)cognise in past perceptions of past phenomena of behaviour and outer appearance.

However, the past and the future are not realities. They are constructions of the human mind. The ancient philosopher Augustine (354-430 AD, 1961) considered linear time perception an achievement of the human mind and its "enduring attention" (Hausheer 1937). Hence, constructs of personality, because they describe patterns over time, require conscious awareness and time perception. Only those phenomena that people can become consciously aware of are able to become the subject matter of their personal constructs. And only those constructs that they are consciously aware of can be socially shared and lexically encoded. However, people cannot consciously perceive all behavioural phenomena that are important and socially relevant in everyday life (Westen 1996). Pheromones (Grammer et al. 2005) and subliminal odours (Li et al. 2007) influence human behaviour, in particular mate selection (Bhutta 2007), but they are not consciously perceivable. Such phenomena are blind spots that likely evade personal and social constructions of personality (Kagan 1998; Uher, Methodological approaches to personality taxonomies: The Behavioural Repertoire $\mathrm{x}$ Environmental Situations Approach - A non-lexical alternative, unpublished).

Recognising temporal patterns requires time perception. Individuals who are unable to perceive time, and thus temporal patterns, cannot (re)cognise individualspecific patterns - and thus cannot (re)construct personality. Babies seem to have no sense of time at all; it develops only slowly during childhood (Fraisse 1964; Piaget 1969). This may hinder young children even after language acquisition from providing reliable reports about their own habitual behaviours or those of others. In adults, subjective time perception generally accelerates with age and differs among individuals (Carrasco et al. 2001; Joubert 1984). It is also flexible within individuals in that, sometimes, time seems to fly or to drag slowly. Individuals may therefore have different perceptions of temporal patterns of individual behaviour and also of (especially artificially modified) outer appearance - and thus vary in how they construct personality differences. 
Of the sketchy details that individuals can perceive through their shifting perceptual lenses (Brunswik 1952), many may enter their minds just briefly. Those that are memorised are not stored as the "pure" perceptions that they were when they entered the person's mind. Instead, perceptions are transformed into, organised with, and integrated with information that is already stored - in constructs and representations that thereby become continuously modified (Kelly 1955). "Experiences are cognitive resultants of past lives" in categorised form (Peirce 1902, CP 2.84). Thinking is making categories that link the experiences of the present moment with those of the semiotically reconstructed past; the construction of the new is bound to and constrained by the reconstructions of the past (Valsiner 2012).

Consequently, individuals do not and cannot access large accumulations of "pure" perceptions of past events. They cannot base the recognition of individual-specific patterns in unbiased ways on large mental "data bases" of perceptions. Processing such enormous masses of information to identify probabilistic, differential, and temporal patterns would perhaps even exceed their cognitive capacities. All the more given that the identification of such patterns is substantially hindered by the fact that, in behavioural and psychological phenomena, within-individual variation often considerably exceeds between-individual variation (Shweder and Sullivan 1990; Mischel 1968; Uher, An integrative meta-theoretical framework for research on individual behaviour in context - situations, populations, species, unpublished). Instead, individuals can access only information that they have already integrated, transformed, and (re)constructed in iterative processes of perception and construction over time. "Recognition is not a simple accumulation of elements, but an integrated whole" (Komatsu 2012 online, p. 3).

For these reasons, the particular phenomena of behaviour and outer appearance and the particular situations in which they are perceived along with the particular individuals, the particular occasions and the particular spans of time that people actually consider when they mentally (re)construct individual-specificity (i.e., personality; Uher, Meta-theoretical foundations of objectivity versus subjectivity in quantifications of behaviour and personality, unpublished) all remain unknown - also to the construers themselves. These mental (re)constructions necessarily suffer from constraints, biases, and errors in perception, memory, and reasoning (Fahrenberg et al. 2007; Gigerenzer et al. 1999; Shweder and D'Andrade 1980).

\section{Genesis of Socially Shared Constructs of Self- and Other-Perception}

The effective ability to communicate with others substantially increases the information that individuals can obtain. Communication (verbal and nonverbal) may direct an individual to focus its perceptual lens on particular details that others have perceived and that they consider important, thereby promoting the socially shared perception of particular phenomena and thus their salience. Verbal communication enables individuals to exchange information also beyond what is available in the present (Uher, What is behaviour? And (when) is language behaviour? A meta-theoretical definition, unpublished). This opens up further avenues to obtain information that is relevant for (re)cognising individual-specific patterns and that may, at the same time, modify the individual's pertinent constructs and representations. Sharing information about 
individuals is obviously so important to human social life that gossiping about whois-doing-what-with-whom comprises up to about two thirds of conversation time (Dunbar 1996) and occurs in all human cultures (Brown 1991). Central to this gossip is information about average individuals and about particular individuals in the social environment. Obtaining this information inevitably entails comparisons among individuals.

\section{Possibilities for Identifying Individual-Specific Patterns}

The rather static phenomena of outer appearance can be perceived in many individuals at the same time and can therefore be compared rather directly between individuals. By contrast, the dynamic and fluctuating nature of behavioural phenomena hinders their simultaneous perception in many individuals - and hence hinders direct comparisons between individuals. Only in particular situations, such as in footraces in which individuals perform exactly the same behaviour spatially and temporally in parallel to one another, do differences between individuals become directly perceivable. But such situations and uniform performances of the same behaviours in spatial and temporal parallelism are not typical of everyday life.

Psychological phenomena, in turn, can generally not be directly perceived in other individuals (Locke 1689; Toomela 2008)-hence, they cannot be directly compared between individuals. This is a crucial point for psychology, especially for personality psychology. Ultimately, how could people identify differences between individuals in phenomena that everyone can directly perceive in just one single individual — in just oneself? The impossibility of perceiving psychological phenomena in others (Locke 1689) also entails that the validity of inferences from others' externalisations, such as from their behaviour (nonverbal and verbal; cf. Uher, What is behaviour? And (when) is language behaviour? A meta-theoretical definition, unpublished), can never be exactly ascertained (cf. Toomela 2011). Any comparisons based on phenomena that potentially mediate the mind-environment connection are necessarily compromised. They are but social conventions (Valsiner 2012). Even if each individual developed his or her own private metric against which that person could quantify his or her own psychological phenomena - there would be no point of direct comparison by which to convert these metrics across individuals.

But people cannot directly compare their own psychological phenomena even within themselves across time. Psychological phenomena are "of maximum uniqueness - they occur each only once, at the minuscule border of the future and the past we construct as the "present" (Valsiner 1998, 2012, p. ix). They are "actualities" (Gillespie and Zittaun 2010, p. 72). Their perception is inevitably bound to the present, which is described as the "here and now" in the field of psychology. (For considerations of the present and its perception, see James 1893, and Le Poidevin 2004, 2011). Comparisons of perceptions of psychological phenomena within the individual would require comparisons with past ones - which, however, have already ceased to be. All that can remain are memorised perceptions (Le Poidevin 2011) that have already been integrated, organised, abstracted, and (re)constructed - the "cognitive resultants of past lives" (Peirce 1902, CP 2.84; Valsiner 2012). Consequently, perceptions of ongoing psychological phenomena can be compared only with (re)constructions of past perceptions. Direct comparison between present and past 
events of psychological phenomena - and thus quantifications of them - are impossible. Attributions of quantitative properties to psychological phenomena therefore must be-and are - fundamentally challenged (e.g., Brower 1949; Michell 1997; 2003; Omi 2012; Rosenbaum and Valsiner 2011).

The actively construing minds of individuals again transcend these impossibilitieslargely unnoticed as they transcend the one-sided gap of the mind-environment connection. Because each individual can directly perceive his or her own psychological phenomena, and constructs relations among them and to his or her own behavioural phenomena, social exchange may promote the assumption that other individuals have similar experiences and constructions as well. Likewise, social exchange may contribute to the awareness that others also experience perceptual gaps. Vice versa, realising the existence of perceptual gaps and the resultant lack of understanding may trigger social exchange and the formation of socially shared constructs (Moscovici 1961; Voelklein and Howarth 2005). These processes may explain why the entirely internal phenomena of the psyche are commonly incorporated into socially shared constructs of personality. These constructs are, in the strictest sense, socially shared constructs of self-perception, but not of other-perception. The latter is possible only for the phenomena of behaviour and outer appearance.

Through social exchange, individuals can also incorporate into their own personal or subjective constructs those that other individuals have subjectively constructed; thereby merging and shaping them in socially shared ways into intersubjective or social representations (Jovchelovitch 2007) or folk concepts (Tellegen 1993). Because everyone constructs the world differently (Kelly 1955), inferring psychological phenomena from the externalisations of other individuals and comparing the inferred phenomena across individuals and with those of one's own psyche is necessarily a collective act. Compromises are required in order to achieve consensus on the particular inferences drawn and on the particular comparisons made. This may lead to different outcomes in different sociocultural communities as the diversity of, for example, parental ethnotheories shows (Pillai 2012). But social exchange and intersubjective compromise need not necessarily resolve contradictions between personal and socially shared constructs. Individuals and semiotic communities can simultaneously maintain competing constructs in terms of cognitive polyphasia (Howarth et al. 2004; Moscovici 1961).

Semiotic systems are elementary for social exchange-lexical symbols in particular. They are essential for making subjectively constructed meanings accessible to others and for intersubjectively exchanging them among construing minds. However, "language is not merely a reproducing instrument for voicing ideas but rather is itself the shaper of ideas" (Whorf 1958, p. 5). The very communication tools that people use to build up and to express their representations already implicitly contain some structures (Lahlou 1996). Language mediates thoughts and enables abstract thinking (Neuman et al. 2012).

A crucial process through which language enables abstraction is hypostatic abstraction in which perceivable qualities (e.g., sweet) are converted into objects (e.g., sweetness; Peirce 1902, CP 4.227). This reification makes perceivable qualities functionally independent of their embodied experience. As objects, these qualities can be associated with signs that are arbitrarily related. These reified qualities can become objects of reflection and contemplation and can be linked to 
various other modes of perception and to connotations (Neuman et al. 2012). Languages therefore contain not only concrete words that refer to directly perceivable phenomena; they also contain abstract words that refer to ideas and concepts that are distant from immediate perception (Vygotsky 1962). Because the concrete references of abstract words cannot be easily traced anymore, these words are loaded with meanings that likely vary across sociocultural and historical contexts (Neuman et al. 2012). Hence, all words have meaning; the meaning of every word is a construct (Vygotsky 1962).

\section{Contextuality of Constructs of Self- and Other-Perception}

Constructs reflect recurring patterns that have been perceived in the world (Kelly 1955). As such, they represent knowledge. Constructs of self- and other-perception represent knowledge about individuals - average and particular ones - and thus about comparisons between them (see above; Westen 1996).

Knowledge is always context-dependent (Jovchelovitch 2007). The knowledge that constructs of self- and other-perception reflect may depend on the context of particular individuals. It can therefore be ideographic and personalistic (Allport 1937; Kelly 1955; Lamiell 2003; Murray 1938; Stern 1935). It also depends on the individuals' stages of development (Toomela 2010a), on their knowledge and educational level (Vygotsky 1962), and on their abilities to reflect about themselves, their world (Omi 2012), and their life histories (McAdams 1985). Different individuals therefore associate standardised items on personality questionnaires with different "fields of meaning" that need not be identical to the interpretations that the researchers have of these items (Arro 2012; Rosenbaum and Valsiner 2011, p. 47). This knowledge may also depend on the context of particular situations (Bandura 1986; Lazarus 1981; Mischel 1968; Rotter 1954) and on the states of mind that individuals may have in particular situations (Omi 2012), such as particular goals and motivations (Biesanz and Human 2010). It may also depend on the context of particular groups of individuals; therefore, reference group effects can be found (Heine et al. 2002).

The knowledge that constructs of self- and other-perception reflect also depends on the context of the particular semiotic system in which it is encoded. Bilingual people therefore describe themselves differently depending on the language they use-even in translated versions of the same questionnaire (Veltkamp et al. 2012). It is not possible to separate a semiotic system from the sociocultural context in which it was developed (Køppe 2012; Whorf 1958). The knowledge that constructs of person perception reflect therefore also depends on the context of the particular culture in which it was developed. Culture is "the inherent core of human psychological functions" (Valsiner 2009, p. 5). The particular patterns and phenomena that particular groups of individuals perceive as salient, that they construct as socially relevant, and that they semiotically encode (cf. lexical hypothesis) is culture-dependent (Church and Katigbak 1988, 1989). "Cultures select a limited range from among the spectrum of [patterns] to encode in their lexicon, and they may select differently. Languages differ not only in the precise ... terms they include (as every translator knows), but more broadly in the aspects ... their vocabularies emphasize" (McCrae and Costa 1997, p. 510; Angleitner et al. 1990). 
Finally, person-related knowledge also depends on the context of the historical era in which it is developed and encoded (Kagan 1998). The socio-cultural norms of interpretation and appraisal of particular patterns of behaviours and of outer appearances may change over time - and with it the pertinent everyday psychological knowledge. Over time, new lexical symbols (John et al. 1988) - and new meanings (Toomela 2010a; Vygotsky 1962) — are created, whereas others become outdated and are no longer used.

The preceding analyses have shown that lexical approaches and assessment methods investigate everyday psychological knowledge, but not the phenomena that this knowledge is about. The philosophy-of-science perspective will now be used to scrutinise the prevailing strategy to explain individual differences-explicitly or implicitly_with assumptions of "traits".

\section{Strategies of Scientific Explanation Based on Trait Concepts}

Over the last century, psychologists have tried to tackle the major task of systematically and comprehensively categorising individual differences in order to develop explanatory theories of personality. "For, we can look at a theory of personality as a specification of the most important individual differences and then as a model of how they come about" (Goldberg 1981, p. 141). To do so, it was necessary to meet three challenges.

The first challenge was to devise suitable strategies for deciding which kinds of individual differences to select for empirical categorisations (Goldberg 1981; Uher 2008a, b, Uher, Methodological approaches to personality taxonomies: The Behavioural Repertoire x Environmental Situations Approach-A non-lexical alternative, unpublished) - a task that seemed "hopelessly complex" (Thurstone 1934, p. 14). The lexical hypothesis suggested that categorising the collective knowledge about individual differences that people had encoded in their natural languages could be a suitable strategy for developing comprehensive taxonomic models of individual differences. The compilations of lexical descriptors in comprehensive lexica-the "storehouses of folk knowledge" (John et al. 1988, p. 174) - that were already available for various languages made this a viable, though labour-intensive approach (Allport and Odbert 1936; Norman 1967).

The second challenge was to devise methods of scientific measurement of individual differences (Uher 2008a). The lexical hypothesis suggested that enquiring about lay people's constructs about individual differences could be a suitable scientific method (Block 2010; Westen 1996). The ease of collecting such data from many individuals made assessments by lay people the "preferred" (Matthews et al. 2003, p. 5) "if not the standard" method (Rosenbaum and Valsiner 2011, p. 50; italics added) on which personality psychology "relied heavily" (Baumeister et al. 2007, p. 396).

The third challenge was to devise methods for the systematic reduction of the complex data sets obtained through assessments, be they based on lexical or other approaches (cf. Uher 2008a; Uher, Methodological approaches to personality taxonomies: The Behavioural Repertoire x Environmental Situations Approach-A nonlexical alternative, unpublished). Many statistical methods have been developed to identify the structures that underlie empirical data to enable parsimonious 
descriptions of their complex manifest structures (e.g., factor analyses; Thurstone 1934; Cattell 1952).

Statistics describe structures that are latent to data. Assessment data reflect how much people believe (i.e., construct) that particular lexical descriptions apply to particular individuals. Lexical descriptions encode the socially shared knowledge about individuals. This knowledge comprises everyday psychological descriptions of patterns of behaviour and of outer appearance as well as potential causal explanations - be they accurate or not - in terms of inferred psychological phenomena in particular. Assessments rely on this knowledge. They also rely on the personal and socially shared constructions about how to quantify - the not directly perceivable - individual-specific patterns in these phenomena (see above). Consequently, the latent structures that can be identified by lexical or other assessment studies reflect structures that underlie the socioculturally shared construction, interpretation, appraisal, and explanation of consciously perceived individual differences (Borkenau and Ostendorf 2008; John 1990). These structures describe categorical systems of personality descriptions in naturallanguage terms (Goldberg 1982; John and Srivastava 1999) and thus comprise models of everyday beliefs about personality (Westen 1996)-i.e., the systems of collective knowledge.

The prevailing psychological interpretation of the structures that are latent to assessment data, however, is that they reflect stable structures that are latent to the individual-specific phenomena that are being described and assessed. Hence, as an analogy to statistical methods in which true (i.e., stable) structures are modelled as latent "traits" that underlie complex empirical (observable) data and that can statistically explain these data, these structures are assumed to reflect stable structures in psychological, physiological, and other phenomena internal to the individual that causally influence and can thus explain that individual's (perceivable) behaviour. These structures are called "traits" or "dispositions" in personality psychology (Allport 1937; Matthews et al. 2003).

The statistical approximation of true scores that are free of measurement error refers to precision in scientific measurement. Measuring personality "traits" that are stable rather than that fluctuate across occasions and across situations, as is the case for behaviour seems therefore intrinsically plausible and scientifically valuable. But this reasoning is based on a fundamental fallacy entailed by the human language.

Language enables abstraction because it allows us to make perceivable qualities independent from their immediate perception by reifying them into linguistic objects (i.e., hypostatic abstraction, Peirce 1902, CP 4.227; see above; Neuman et al. 2012). Reifications are prone to the fallacy of misplaced concreteness in which abstractions are treated as concrete (Whitehead 1929). The idea of individual-specific patterns in behavioural and other phenomena requires high levels of abstraction. The linguistic reification of this complex and highly abstract idea through lexical encodings, such as by the terms "traits" or "dispositions", is therefore inherently prone to this fallacy. It misleads people to treat "traits" and "dispositions" as concrete actual entities.

The reification of constructs of self- and other-perception as causal entities that underlie the phenomena that are being perceived and constructed are widespread in everyday psychology. For example, individuals who behave aggressively at some point in time are ascribed as having aggressive "traits" or "dispositions"; and the construction of them as having these "traits" is used to explain the fact that they 
behaved in this manner in a given moment. In everyday life, linguistic reification facilitates and accelerates the exchange of information, despite its inexactitude. It may therefore disseminate more quickly than more accurate yet cumbersome descriptions. Everyday psychology is therefore full of reifications and circular explanations (Laucken 1974).

The assumption of "traits" as heritable structures internal to the individual (Brody 1994) that "determine behaviour in a defined situation" (Cattell 1950, p. 222) ascribes to these abstract ideas not only a concrete existence, but furthermore a causal status (Bock 2000). But assessments of "traits" do not and cannot capture causal entities inside the individuals assessed (Komatsu 2012). "Traits" are lexically encoded and socially shared constructs about recurring patterns in phenomena perceivable in the assessed individuals - i.e., in behaviours and in outer appearances. As such, "traits" are categorical summary statements about a person's behaviour (Wiggins 1979). Using these summary statements to explain and predict behaviour means "in effect using a description of behaviour to explain it" (Mischel and Shoda 1994, p. 157).

The circularity of psychological explanations that are based on trait concepts may not be as directly apparent as that of the explanations that are provided by everyday psychology. Scientific constructs of "personality traits" are the result of long and highly complex series of abstraction processes. The phenomena to be explained are first filtered through iterative and repeated processes of perception, personal and socially shared (re)construction, lexical encoding, and assessment by lay psychologists (see above). The outcomes of these processes are then further condensed by the application of complex statistical methods by scientific psychologists. The complexity of these processes of abstraction, of which the everyday psychological ones are still poorly understood (Rosenbaum and Valsiner 2011), may mask the circularity of these explanations - but cannot overcome it.

In a nutshell, psychological explanations based on trait concepts equate the statistical structures underlying the assessments of encoded constructions about perceivable phenomena with the phenomena that underlie these perceivable phenomena. This explanation is circular at a very high level of abstraction and complexity, but it is essentially circular. Figure 2 illustrates this schematically. Based on the erroneous assumption that $\mathrm{D}$ is a measure of $\mathrm{C}$, which underlies $\mathrm{A}$, it is concluded that $\mathrm{D}$ measures what underlies A and that it can thus causally explain A. But meta-theoretical analyses show that D can only be the latent structure of B, which are the constructs and representations that people develop of A. Explaining A with D is thus circular.

Given that assessments reflect socially shared knowledge about recurring patterns in behaviour and outer appearance, it is not surprising - but rather to be expectedthat "personality predicts behaviour" (Paunonen and Ashton 2001) and that, vice versa, individuals can use "thin slices of behaviour" (Borkenau et al. 2004), "minimal appearance cues", such as "facial structures" (Kramer and Ward 2010) or shoes (Gillath et al. 2012) to activate this knowledge and use it to "accurately assess others' personality" even at zero acquaintance (Shevlin et al. 2003). But such cues are not "signals" of entities internal to individuals (Kramer and Ward 2010) that people are able to decipher. These findings merely reflect the socially shared knowledge about how particular individual-specific patterns in behaviour and outer appearance typically go together in a particular semiotic community and how that community (causally) explains this. This knowledge may also be reflected implicitly 


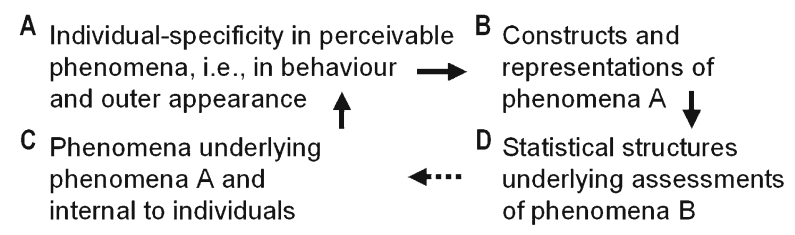

Fig. 2 The fundamental circularity of psychological explanations based on trait concepts. Note. Based on erroneous meta-theoretical assumptions about what phenomena are actually reflected by assessment data, D is considered to be a measure of $\mathrm{C}$, which are the phenomena that underlie A. Therefore, it is concluded that D measures what underlies A and that D can thus causally explain A. But D can be the latent structure only of the phenomena B, which are the constructs and representations that people develop of A. Thus, explaining A with D is circular

in lexical descriptors that do not refer explicitly to outer appearance (which were excluded from lexical approaches). Likewise, it is also to be expected that personality assessments are stable across decades (Conley 1984; Soldz and Vaillant 1999). This stability is also a property of the socially shared knowledge they reflect.

At the bottom of psychological explanations based on trait concepts is the - anything but new - insight that "past behaviour is the best predictor of future behaviour". It may be circular to predict behaviour with behaviour-and this is why assumptions of independent internal entities are so tempting. But it is not circular to predict future events from past events, in particular not in phenomena as dynamic and fluctuating and inherently bound to the present, such as those of behaviour. In complex dynamic environments, such as in the social world, identifying recurrent patterns in these phenomena can provide orientation and predictive control. But prediction is not yet an explanation. Past behaviour can be causally related to future behaviour, though only indirectly, such as through the mediation of environmental (in particular social) transactions or through the knowledge that people have developed about it. But this cannot explain how behavioural patterns emerge in the first place. The direct causes of behaviour, which are primarily sought in psychological, biological, and other internal phenomena as well as in external environmental conditions, cannot be captured through assessments and causal reifications of behavioural descriptions. They can be explored - in themselves and as possible causes of individual behaviour - only if they are studied in their own rights (see below).

\section{Implications for Personality Psychology}

The circularity of scientific explanation based on trait concepts derives from the fundamental challenges that the different kinds of phenomena entail that are described in the lexical hypothesis. Of the phenomena that people construct and represent, many are bound to the present, such as those of behaviour and the psyche, which makes direct perceptions of individual-specific patterns impossible. Their (re)cognition requires repeated perception, memorisation, and mental reconstruction (Uher, Meta-theoretical foundations of objectivity versus subjectivity in quantifications of behaviour and personality, unpublished). But the dynamics and fluctuations of these phenomena - and the individual's narrow perceptual lens filters that shift across these phenomena's fluctuating events - enable only fragmentary and 
inconsistent perceptions (Brunswik 1952), which thus hinders the (re)cognition of individual-specific patterns. Nonetheless, to enable orientation and sense of control, human minds transcends these gaps (Daston and Galison 2007; Valsiner 2012) and reify - promoted through language (Neuman et al. 2012) - mental (re)constructions of the perceived into causal entities (Bock 2000; Lamiell 2003), thus blending a phenomenon's description with its explanation (Mischel and Shoda 1994). This obscures the vital differentiation between the phenomena to be explained, the explananda, from those explaining them, the explanantia (Hempel and Oppenheim 1948; Popper 1934) — and inevitably entails explanatory circularity.

These are challenges to everyday psychologists (Laucken 1974) and scientific psychologists alike. The latter are always both. "All scientists are victims of a linguistic structure that partially controls our thinking" (Howard 1994, p. 400). Underlying this structure are the basic assumptions that their particular semiotic communities maintain about the "lived" relations between individuals and their world-i.e., their ideologies (Althusser and Balibar 1970, p. 314). Ideology precedes science; it "constitutes the prehistory of a science" (p. 45). By breaking radically with the epistemological frame of reference of prescientific (i.e., ideological) notions and by constructing new patterns through scientific methodology-i.e., through thinking about phenomena and the means of producing knowledge [German: Wissen schaffen] about them-science [German: Wissenschaft] is produced. But alongside science, ideologies survive as essential elements of every social formation (Althusser and Balibar 1970). Sciences are always embedded in the sociocultural ideologies that operate through the meanings that semiotic communities attribute to their activities through common sense (Valsiner 2012).

This may explain why scientific psychologists - at least in Western societies, where basic assumptions of homo rationalis (Simon 1993) prevail—are so confident about the abilities of everyday psychologists to comprehensively identify, accurately describe, and validly quantify all individual differences that are socially relevant and important in everyday life - as this is prominently reflected in the lexical hypothesis and its fundamental significance as one of the most important-if not the most important-guiding theoretical assumptions in modern personality psychology (cf. Ashton and Lee 2005).

The Current State of Knowledge about the Lexical Hypothesis

Despite its enormous importance, the lexical hypothesis itself has not yet been made an object of scientific inquiry. Still today, almost 130 years after its first articulation (Galton 1884), it has remained but a hypothesis. To gain a more comprehensive understanding of this hypothesis and its current state, it is important to explore its possible origins.

The lexical hypothesis was not derived from a specific theory; rather, some specific theories were developed based on parts of its basic statements and on the results obtained from empirical applications of it (Goldberg 1981). Most likely, the lexical hypothesis was generated by abduction - a form of logical inference that Peirce (1901, CP 7.218) introduced as "guessing" and considered "merely preparatory". Abduction is an essential epistemological step in scientific reasoning. It begins with surprising facts that are in need of an explanation that is not available at the 
outset. Consideration of the surprising facts suggests a hypothesis that, if it was true, would explain the observed facts as a matter of course. The particular hypothesis is suggested by the resemblance between the facts and the consequences of the hypothesis if it is found to be true. "Hence, there is reason to suspect the generated hypothesis is true" (Peirce 1903, CP 5.188-5.189). But its truth is still uncertain because abduction seeks to explain facts ascertained in the present by inferring possible causes of these facts, which necessarily lie in the past and thus have already ceased to be (Valsiner 2012).

For Galton (1884), it may not have been a single surprising fact that led him to abduce the lexical hypothesis, though the richness of lexical descriptors about individuals could indeed surprise someone who is unfamiliar with human languages. But rather, it may have been the reduction problem of a science that is concerned with the surprising complexity of the real phenomena of individual differences. If the lexical hypothesis was true, lexical descriptors of individual differences would be self-evident consequences. Thus, some of its statements could provide an explanation for the existence of the pertinent repertoires in everyday languages and-based on this - a possible solution to the reduction problem of personality psychology.

Once hypotheses are generated abductively, they are used as general premises to reach specific conclusions in scientific reasoning. Two approaches to argumentation are thereby possible: deductive and inductive. If the truth of the general premise is believed to definitely establish the truth of the conclusions, then the argument is deductive. If the truth of the general premise is not assumed to definitely establish the truth of the conclusions, but to provide good reasons to believe that they are true and unlikely to be false, then the argument is inductive. Thus, deduction seeks a logically certain conclusion that is derived from the premise, whereas induction seeks facts that support or falsify the premise. But neither deduction nor induction can leave the realm of analysis that the hypothetical premise defines and delimits. They cannot create new knowledge. This is possible only for abduction because it "seeks a theory" (Peirce 1901, CP 7.218). Hence, abduction creates new knowledge-yet at the expense of a priori certainty about its truth. This uncertainty allows - and intrinsically calls - for their constant reconsideration and reconstruction (Valsiner 2012; cf. also Althusser and Balibar 1970; Weber 1949).

For the lexical hypothesis, this has not yet been done. Personality psychologists took this hypothesis as a true premise by which to reach the specific conclusion that the human lexical repertoires constitute finite sources of information for categorising individual differences (Allport and Odbert 1936; Cattell 1943; Goldberg 1993; John et al. 1988). But inductive investigations have not yet been done; personality psychology did not-and still does not - seek facts that may support or falsify this hypothesis. It is still unknown whether in fact all individual differences that are important in interpersonal life are lexically encoded as stated by the lexical hypothesis. For example, how well are individual differences in behaviours that are not consciously perceivable yet are known to influence social behaviour (see above) represented in everyday psychological knowledge? And how is this knowledge lexically encoded? Where do the differences in taxonomic models come from that have been derived from different languages? Do they reflect actual differences in the individual-specific behaviours and outer appearances between different language communities, just differences in what these communities consider salient and socially 
relevant or in how they interpret and appraise what they perceive, or all of these together (see below)?

The status of the truth of the lexical hypothesis is still as uncertain as it was at the time of its abduction almost 130 years ago (Galton 1884). Even worse, at this historical point in time, personality psychology is de facto unable to test this hypothesis empirically because its basic statements have been only partially considered so far. Psychologists have so far systematically categorised only people's lexically encoded constructs and representations, but they have not even tried to achieve this for those phenomena that are constructed and represented in everyday life - that is, for individual-specific patterns in behaviour (Uher, Methodological approaches to personality taxonomies: The Behavioural Repertoire x Environmental Situations Approach-A non-lexical alternative, unpublished) and in outer appearance. Psychologists have revealed only half of the story of personality so far. However, comprehensive categorisation systems of individual-specific behaviours and appearances are needed to unravel how lexically derived models actually represent perceivable individual differences; the ways in which they may reflect different perceptions, interpretations, and appraisals; and how individual behaviours and outer appearances actually vary within and among different sociocultural and language communities (cf. Block 2010). This knowledge is also essential for systematic investigations of the actual causal mechanisms behind many fascinating findings that have been found by using lexically derived taxonomic models. Ten desiderata for future research are specified below.

\section{Lack of an Elaborated Philosophy of Science for Personality Psychology}

As scientists concerned with phenomena that are important in each individual's everyday life, personality psychologists are particularly susceptible to the "psychologist's fallacy" (James 1893, p. 196) - the unwanted confusion between our own personal standpoints and the scientific objects we study. "It is true that in our sciences, personal value-judgments have tended to influence scientific arguments without being explicitly admitted" (Weber 1949, p. 54). "Psychology is a science which is peculiarly liable to distortion by pseudo-scientific, political and religious intrusions" (Cattell 1950, p. 11). Like all individuals, researchers have socioculturally guided minds (Valsiner 1998). Ideologies about the relations of individuals - average and particular ones - to their social world - i.e., about personality - are particularly pronounced. The influences of such ideologies on the scientific practices in personality research are therefore particularly profound and difficult to recognise and to confine.

Scientific ideas should be separated from the sociocultural ideologies into which they are embedded and that all individuals as members of their particular semiotic communities have in their minds (Valsiner 1998). Unfortunately, an explicit and elaborated philosophy-of-science framework that could help personality psychologists to continuously rethink and challenge their own ways of generating knowledge has not yet been established-despite Rychlak's (1968) promising start half a century ago and apart from brief sections on ideographic and nomothetic approaches and discussions of the "images of man" of major schools of thought contained in many textbooks. But philosophy-of-science tools are essential for regularly rethinking and 
challenging the meta-theoretical structures that have became established in schools of thought and in the scientific practices of a discipline (Kuhn 1976).

Nature is vastly too complex to be explored even approximately at random. Something must tell the scientists where to look and what to look for, and that something, though it may not last beyond his generation, is the paradigm with which his education as a scientist has supplied him. Given that paradigm and the requisite confidence in it, the scientists largely ceases to be an explorer at all, or at least to be an explorer of the unknown. Instead, he struggles to articulate and concretize the known. (Kuhn 1976, p. 61)

Paradigmatically fixed and no longer challenged assumptions close the doors to intellectual innovation (Valsiner 2012). In personality psychology, the paradigmatic establishment of trait concepts has hindered elaborations and explications of the meta-theoretical assumptions that underlie this particular concept and the school of thought in which it was developed, which has impeded the recognition of its fundamental explanatory circularity. But meta-theoretical assumptions are always effective in any scientific practice - even if they may be effective only implicitly. The assumption that the "description of personality must precede, not follow, personality theory" (Costa and McCrae 1992, p. 861) overlooks the methodological matter of fact that all empirical facts are defined as facts only based on particular theoretical assumptions.

At the same time, the dogmatic and unquestioned status of assessments by laypeople as the standard methods of "scientific measurement" has hindered the explication of the meta-theoretical basis on which they rest. Selecting methods a priori rather than purposefully for given questions narrows researchers' perceptions of real phenomena and blinds them to particular elements (Gillespie and Zittaun 2010; Omi 2012; Toomela 2011; Westen 1996). The tempting assumption that assessments constitute a "direct approach to find out what people think or feel" by using a "simple and straightforward task" (Rosenbaum and Valsiner 2011, p. 50) that requires only "abbreviated introspection" (p. 47) has blinded many psychologists to the fact that the phenomena of the psyche and of behaviour are inherently bound to the present (Gillespie and Zittaun 2010; Uher, What is behaviour? And (when) is language behaviour? A meta-theoretical definition, unpublished; Valsiner 1998, 2012) and cannot be captured by retrospective assessments at all (Uher, Metatheoretical foundations of objectivity versus subjectivity in quantifications of behaviour and personality, unpublished).

Their efficiency with regard to data collection in large samples in conjunction with sophisticated statistical methods for data analysis has given assessment methods a scientific shape that has consolidated their status as the standard empirical method (Omi 2012; Sato et al. 2010; Toomela 2011; Westen 1996). But this a priori status has precluded an enquiry as to what the phenomena actually are that are being converted into data in assessments - and thus has precluded a rethinking of how these data can be analysed and interpreted at all (Gillespie and Zittaun 2010; Køppe 2012; Toomela 2011; Valsiner 2012). This has hindered many from recognising that assessments can reflect only knowledge structures, but not past events of psychological or behavioural phenomena. It has also hindered the recognition that, in the fluctuations of behaviour, 
individual-specific patterns cannot be directly perceived and thus cannot be directly quantified. The a priori status of these methods has also hindered the recognition that psychological phenomena may not - and actually cannot - have quantitative properties that could be "measured psychometrically" because they, too, are phenomena that are inherently bound to the present (Brower 1949; Michell 1997, 2003; Omi 2012; Rosenbaum and Valsiner 2011; Schwarz 2009; Trendler 2009; Valsiner 2012) and that therefore, they cannot be compared with one another directly.

The lack of an elaborated meta-theory has also hindered many personality psychologists from considering the fact that analyses of individual assessments on the basis of their between-individual variation cannot yield results that are generalisable to the understanding and explanation of within-individual variation - much less of individual development or of the dynamics of internal mechanisms (Cervone et al. 2001; van Geert and van Dijk 2002; Omi 2012; Molenaar 2004a, b). The particular numbers of dimensions of individual differences and their compositions are not natural entities to be "discovered" anyway. They depend on the meta-theoretical decisions made by scientists with regard to how real phenomena can be conceptually reduced to scientific phenomena as well as their decisions about how the thus-derived data can be reduced statistically (cf. Block 2010; Lamiell 2003). Statistics such as factor analyses are not "de facto theories" (Gigerenzer 1991) or "mechanical truth generators" (Meehl 1992, p. 152). Rather, they are but "mathematical thinking tools" (Valsiner 2012, p. 201; Loftus 1996).

The focus of personality psychology on trait concepts, lexical encodings, and assessment methods has hindered the ability of researchers to elaborate the metatheoretical assumptions on which the lexical hypothesis is based. Personality psychologists have therefore failed to recognise that the hypothesis explicitly refers to different sets of phenomena of which they have systematically explored only lexically encoded representations so far, but have seriously neglected the phenomena that are represented. Assumptions that "personality and its assessment are intimately bound with natural language" (McCrae and Costa 1997, p. 510) and that "the idea of personality traits may be as old as human language itself" (Matthews et al. 2003, p. 3 ) clearly refer to personality as the lexically encoded structures of everyday psychological knowledge. But these structures cannot be understood and explained without researching the phenomena that they are about.

The Dialectical Interplay Between Encodings, Constructs, and the Phenomena Being Constructed

Individual-specific patterns of behaviour can also be found in babies and preverbal children (e.g., Kagan et al. 1988) as well as in many other species (Allport 1937, p. 24). Among these species are those that are closely related to humans, such as nonhuman primates (Uher et al., Contextualised behavioural measurements of personality differences obtained in behavioural tests and social observations in adult capuchin monkeys (Cebus apella) unpublished), but also some that are related only remotely, such as octopuses (Sinn and Moltschaniwskyj 2005). Hence, individualspecific patterns in behaviour (and in outer appearance; Darwin 1859) are phylogenetically much older than the human semiotic systems that describe such patterns. Ancestral humans initially developed lexical and other symbols to refer to something 
that they had perceived, (re)cognised, and mentally and socially constructed-and that had thus already been there. Physiology, the psyche, and behaviour develop(ed) before language - both in ontogeny and in phylogeny.

It would be fascinating and insightful to study individual human ancestors who existed before humans had developed the faculty of language. Direct evidence of when humans started to develop socially shared constructs of individual-specific patterns of behaviour and outer appearance and pertinent semiotic symbols in phylogenetic history is lacking. But indirect evidence comes from Palaeolithic dog fossils that suggest that canine domestication had already begun at least some 30,000 years ago (Germonpré et al. 2009) and from an impressive 40-year breeding experiment in another canine species. In farm foxes, strong selective breeding solely for low fearfulness of and low aggressiveness to humans (i.e., tamability) over only 30-35 generations entailed a host of changes in genes, morphology, physiology, and behaviour in which the present day's domesticated species differ markedly from their wild relatives. These findings suggest that individual-specific patterns in behaviour rather than size or reproductive capacity have been the key factors of artificial selections that humans have imposed on some species during domestication (Belyaev 1969; Trut 1999). Hence, humans must have had been able to mentally construct and socially represent individual-specific patterns in behaviour in some nonhuman species already tens of thousands of years ago. Very likely, pertinent representations and semiotic symbols referring to their conspecific individuals had already been developed before.

Individual members of many nonhuman species also develop representations, such as of their environment (Tolman 1948). In some species, in particular those with complex social systems, individuals also develop socially shared representations as reports on community formation and intercommunity warfare in chimpanzees suggest (Goodall 1986; Standford 1998). Individual members of some animal species develop representations that are socially shared even with humans, among them bonobos (Savage-Rumbaugh and Fields 2000), gorillas (Patterson and Linden 1981), dogs (Kaminski et al. 2004), and grey parrots (Pepperberg 2002). But none of these species have developed complex semiotic systems on their own so far. Without pertinent semiotic systems, however, the development and propagation of socially shared representations is substantially impeded.

Whether some nonhuman species are able to recognise and mentally construct individual-specific patterns of behaviour among their conspecifics is still unknown. Evidence of individualised dyadic relationships among nonkin (e.g., in Rhesus macaques; Weinstein and Capitanio 2008, 2012) suggests some degrees of pertinent abilities at least in nonhuman primates. But more research on the abilities that are prerequisites for such abilities (e.g., perception of fluctuating phenomena, perception of time, see above) is needed. For example, many animal species perceive their environments in far greater detail than most humans are able to (Grandin and Johnson 2005), such as chimpanzees that have been shown to have eidetic working memories (Inoue and Matsuzawa 2007). Brunswik's (1952) concept of narrow perceptual lens filters shifting flexibly across the environment may not apply well to them. On the one hand, these abilities may allow these nonhuman species to perceive individual behaviours more accurately than humans. But on the other hand, this massive amount of information and their more limited capacity for information 
processing and long-term memory may hinder their ability to recognise and construct individual-specific patterns in these perceptions (see above). Research on the pertinent abilities of humans who have eidetic perceptual or memory abilities (e.g., some people on the autistic spectrum) can provide important insight in this regard.

But perhaps nonhuman species' lack of language or of a similarly efficient semiotic system to exchange information about individuals and to enable abstract thinking (Peirce 1902, CP 4.227; Vygotsky 1962) does not allow them to construct individual-specificity in the highly abstract form that humans are able to. Individualised relationships may indicate that individual members of some species are able to construct that some specific others show particular behavioural regularities towards them, which may allow some predictions of their future behaviour. But they may perhaps not be able to construct that individuals in their social environment generally differ from one another in particular ways without referring to specific ones. Constructs of personality differences enable humans to quickly assess strangers (McAdams 1994) based on category systems that have proven to be socially significant within the community. Along with the ability to form impressions of others rapidly, which may have co-evolved dialectically, this allows people to gain some cognitive control of interactions with unknown individuals (cf. Goldberg 1981). Maybe the ability to construct personality differences is an essential cognitive tool that enables humans to deal with anonymous others, which seems to be a uniquely human ability (cf. Blaffer-Hrdy 2009) at least among mammalian species. This hypothesis shall be called the personality-constructs-promote-peaceful-anonymouscontacts hypothesis. It suggest that ancestral humans must have developed these abilities at least when they began dealing with unknown individuals outside their particular community on a more regular basis, such as for peaceful traffic, exchange, and trade, for which direct archaeological evidence exists.

Humans of the present day are born into a world full of complex semiotic systems and social representations. Individuals' own perceptual constructs, those that are socially shared within their sociocultural community, and their lexical encodings therefore develop in tight dialectical interplays with one another and cannot be disentangled anymore (Lahlou 1996, 2001, 2008). They also develop in dialectical interplays with the individual-specific patterns in behaviours and in outer appearances to which they refer. Individuals like to belong and therefore adjust their behaviours in order to socially conform. At the same time, they like to set themselves apart as individuals within their communities and to stress their individual-specificity-both in behaviour and in an artificially modified outer appearance (though to different degrees in different sociocultural communities). The possibility for semiotic exchange about individual differences may therefore have promoted individual diversification in humans - as compared to other species that cannot directly communicate about their perceptual constructs at the level of their constructed meaning. Instead, they each have to develop these constructs individually based on their own perceptions and interpretations of what they perceive. Consequently, the development of semiotic systems could have been a driving force for individual diversification during human phylogeny. This fascinating hypothesis, which shall be called the language-promotes-individualisation hypothesis, can be investigated only if research methodology is substantially enlarged and diverse species are studied (see below). 
For modern personality psychology, semiotic exchange entails additional important implications. Through semiotic exchange, scientific knowledge also becomes disseminated to the public. Many scientific concepts - some as old as Galens's four temperamental types, others as scientifically contentious as Freud's personality theory-have become established parts of everyday psychological knowledge. How scientific concepts disseminate in everyday psychology was studied in psychoanalytic concepts and their dissemination among the French public in the 1960s (Moscovici 1961). Everyday psychologists learn about scientific developments through the public media, education guidebooks, and popular science books. The interest in personality is particularly lively given its importance in each individual's everyday life. Terms originating from science, such as melancholic, choleric, extraverted, neurotic, or psychotic, are widely used in everyday psychologycorrectly or not-as are the ideas that childhood experiences and genetic inheritance influence adult individual behaviour. The body of everyday psychological knowledge is not free of scientific knowledge anymore as it may have been in prehistoric times.

This has far-reaching implications for scientific personality psychology. If lexical descriptions - in lexical approaches and in assessments - continue to be the primary source of information for the scientific study of personality, this not only triggers further explanatory circularity, but also leads to a growing convergence between everyday psychological and scientific constructs of personality. As a result, scientific concepts of personality increasingly appear to "explain" lexically encoded individual differences better - in particular because (psychology) students comprise important parts of empirical samples. Such findings suggest progress in science-when in fact scientific understanding of the described phenomena has not necessarily advanced. John B. Watson's and Sigmund Freud's theories of personality development had profound (and fairly different) impacts on educational practices and on everyday explanations of individual behaviour (Wiggins 2003). These practices and explanations in turn may have influenced the individuals' behaviours themselves - though not necessarily in the ways postulated by these theories.

The strong focus of personality psychology on human language, be it through lexical research or assessment methods, has hindered these illuminating insights. The ancient philosopher Plato (428-348 BC) had already recognised that "one should leave the study of words behind to investigate the realities expressed through them" (Politeia, 438d2-439b8, cited in Bordt 1998, p. 156; translated). This is essential because, when words are studied instead of real phenomena, criteria for judging empirical results cannot be established because there will be only many different names or descriptions for phenomena, but no way to decide which descriptions - if any at all—should be accepted (Toomela 2011). Personality psychology should finally explore the fascinating phenomena with which it concerns itself in their entirety and from much broader perspectives than previously done.

\section{Ten Desiderata for Future Research}

It is not important that we better understand which particular behaviours socially shared knowledge about personality allows us to predict or which minimal cues of 
whatever kind can activate this knowledge because these questions are intrinsically circular (see Fig. 2). Studying global structures of everyday psychological knowledge without setting them in relation to the particular contexts on which they depend is also not very important to do. The "discovery" of five major dimensions has provided merely a snapshot of the everyday psychological knowledge that particular semiotic communities had encoded about individuals in particular sociocultural environments and in particular historic times.

It is essentially important, however, to fully consider in their entirety the metatheoretical assumptions that underlie the lexical hypothesis, to systematically test the hypothesis using inductive approaches, and to move personality psychology beyond this particular hypothesis to create new knowledge. Ten desiderata are thereby primary.

Desideratum 1: A Philosophy-of-Science Framework for Personality Psychology

A comprehensive philosophy-of-science framework that explicitly incorporates seven central issues ( $a-g$ in the following) should be developed.

a) Meta-theoretical definitions of the scientific phenomena constructed as personality, i.e., of individual-specific patterns in behaviour and outer appearance and of the everyday psychological knowledge about such patterns, including specifications of the principles and conditions for their demarcation from real phenomena and specifications of their ontology. For individual-specific patterns in behaviour, such a meta-theoretical framework has already been elaborated (see above, Uher 2011a) and successfully applied in empirical studies on capuchin monkeys (Uher et al., Contextualised behavioural measurements of personality differences obtained in behavioural tests and social observations in adult capuchin monkeys (Cebus apella), unpublished) and great apes (e.g., Uher et al. 2008). This framework comprises principles for defining behavioural phenomena (Uher, What is behaviour? And (when) is language behaviour? A meta-theoretical definition, unpublished) and for defining entities (i.e., categories) among their qualitative elements (Uher, Meta-theoretical foundations of objectivity versus subjectivity in quantifications of behaviour and personality, unpublished). It explicitly considers both within- and between-individual variations in the contexts of situations, within-species populations (e.g., cultures), and species using ipsative-normative-populative-speciative approaches (Uher, An integrative meta-theoretical framework for research on individual behaviour in contextsituations, populations, species, unpublished). To differentiate between the different sets of phenomena to which the lexical hypothesis refers, the framework relies on two meta-theoretical kinds of taxonomic personality constructs - one for the phenomena of encoded knowledge and one for the phenomena that this knowledge is about (i.e., scientific-statistical second-order and first-order constructs; Uher, Meta-theoretical foundations of objectivity versus subjectivity in quantifications of behaviour and personality, unpublished). Corresponding frameworks for individual-specific patterns of outer appearance and for the pertinent everyday psychological knowledge still have to be elaborated. The development of these frameworks can capitalise on the meta-theoretical 
elaborations of individual-specificity in the dynamic and fluctuating phenomena outlined above, but should also draw on developments made by other disciplines that study physical appearance (e.g., research on kin recognition and attractiveness) and language and knowledge systems (e.g., psycholinguistics, semiotics, computational linguistics).

b) Questions that can be asked about the thus-defined scientific phenomena. Tinbergen's (1963) four key questions about the causation, ontogeny, adaptivity, and phylogeny, originally formulated for the species-level study of behaviour, constitute meaningful directions also for the individual-level study of behaviour (Uher 2008a, 2011a) and outer appearance. They can also be applied in part to study the systems of everyday psychological knowledge about individuals, the systems of their semiotic encodings, and the sociocultural and historic variations in these systems.

c) Methodological approaches to decide which elements of real phenomena to select for scientific studies. The methodological approaches previously used to guide the selection of elements of everyday psychological knowledge and of behaviour have already been analysed for their underlying meta-theoretical assumptions (Uher 2008a, 2011a, b; Uher, Methodological approaches to personality taxonomies: The Behavioural Repertoire x Environmental Situations Approach-A non-lexical alternative, unpublished). These approaches can be categorised into different classes depending on their selection rationales (e.g., manifest system approaches, top-down/etic approaches). These rationales build on two meta-theoretical selection principles (i.e., content-based and strategybased) that differ in their ability to reduce the influence of the researchers' own everyday psychological ideas about personality in the very process of element selection. Lexical approaches, for example, are manifest system approaches that base element selection on scientifically reduced (i.e., manifest) language systems. Their rationale formulates a selection strategy that demarcates all persondescriptive words in the lexica as the universe of elements without suggesting the study of any particular ones.

To establish comprehensive taxonomies of individual-specific behaviours, a new manifest system approach that is grounded in the above-mentioned metatheoretical framework (Desideratum 1a) - the Behavioural Repertoire $\mathrm{x}$ Environmental Situations Approach (Uher 2008a, b, 2011a, b; Uher, Methodological approaches to personality taxonomies: The Behavioural Repertoire x Environmental Situations Approach-A non-lexical alternative, unpublished) - has been developed. This approach bases element selection on the behavioural-ecological systems of a population or species as far as these have already been scientifically reduced (i.e., are manifest). It formulates a systematic strategy to generate constructs and select their operationalisations based on the established scientific descriptions of the average individual's behaviours and of the environmental situations in which these typically occur. In line with the philosophy of science outlined above, these constructs and the taxonomic constructs that can be empirically derived from them are descriptive; they are not ascribed an a priori causal status. As is true for any research, this approach necessarily relies on language. But in contrast to lexical approaches, it is not guided by the lexical encodings of lay people's representations in which 
descriptions of behaviour are often blended with interpretations, appraisals, and explanations and loaded with implicit sociocultural meanings. This new approach has already been successfully applied in studies on capuchin monkeys (e.g., Uher et al., Contextualised behavioural measurements of personality differences obtained in behavioural tests and social observations in adult capuchin monkeys (Cebus apella), unpublished) and great apes (Uher 2011b; Uher et al. 2008) in which it yielded substantial empirical evidence for individual-specific patterns in behaviour not previously described by other approaches, including those derived from human everyday language. Corresponding methodological approaches for taxonomising individual-specificity in outer appearance should be developed by capitalising on the expertise of other disciplines (cf. Desideratum 1a).

d) Methods of investigation describing a portfolio of diverse techniques of how the selected elements of real phenomena can be converted into quantifiable entities and encoded as data, including the meta-theoretical assumptions underlying these conversion decisions. The meta-theoretical assumptions underlying these decisions in investigations of individual-specific patterns in behaviour were already elaborated using set-theoretic considerations (Uher, Meta-theoretical foundations of objectivity versus subjectivity in quantifications of behaviour and personality, unpublished). They include principles of how the quantitative properties of qualitatively defined entities (cf. Desideratum 1a) can be encoded as data based on established ethological techniques (e.g., Lehner 1998). This allows for the explicit and systematic definition of all considered elements of the set $B$ of behaviours, the set $S$ of situations that functionally mediate these behaviours, the set $T$ of occasions and spans of time in which they are measured, and the set $I$ of individuals in which they are studied. The interrelations of these elements within and among these four sets can therefore be analysed for individual-specific patterns using ratio-scaled measurements in set-theoretically objective ways (Uher, Meta-theoretical foundations of objectivity versus subjectivity in quantifications of behaviour and personality, unpublished). Principles for establishing functional and structural comparability of thus-derived taxonomic constructs of individual-specificity furthermore allow for set-theoretically objective comparisons between populations (e.g., cultures) and species (Uher, An integrative meta-theoretical framework for research on individual behaviour in context-situations, populations, species, unpublished). Similar principles should be elaborated for investigations of individual-specific patterns in outer appearance by drawing on the pertinent expertise of other disciplines (cf. Desiderata 1a, 1c).

In assessment-based investigations, the encoding of qualitative and quantitative properties of the selected elements into data largely relies on implicit decisions by lay people. The particular elements of the sets $B, S, T$, and $I$ that people consider in their representations and assessments are thus ill defined, as are the elements of the sets of interpretations, appraisals, and explanations that these may comprise. Everyday psychological knowledge should therefore be analysed by using qualitative methods (e.g., Diriwächter et al. 2004; Rosenbaum and Valsiner 2011) and lexical analyses of texts, conversations, and reports obtained in everyday life (e.g., with methods of computational linguistics; Bolden and Moscarola 2000) that allow for the explication of the conversion and encoding decisions made. 
e) Meta-theoretical principles for the reduction, analysis, and theoretical interpretation of data, including the formal-logical operations that are enabled by the encoded phenomena to explore possible causal relations and internal systematics. These principles should be elaborated and matched purposefully to the metatheoretical properties of the phenomena that are reflected by the data (see Desiderata 1c and 1d; Toomela 2011). For example, the mathematical algorithms of the statistical techniques used to develop taxonomic models capitalise on redundancies in the information encoded by the analysed variables (e.g., factor analysis). Lexical factor models thus reflect individual differences for which many synonyms or near-synonyms exist, but they likely miss out on those that are not redundantly expressed in everyday language (Block 2010). This may impose serious threats to the representativeness of lexical factor models because "it is not always the case that the number of synonyms for a word testifies to the psychological importance of that word. Certain words - crucial words - do not need to be redundantly expressed" (p. 3). Important interpersonal perceptions may instead be encoded in idioms and proverbs, rather than in single-word terms.

This is an important issue for any kind of taxonomic research that is based on statistical methods. Assessment tools are developed by selecting variables that yield data with high internal consistency and that thus measure the same concept-i.e., redundancies (Block 2010). But in behaviour, redundancies may be rare and constrained by evolutionary processes (Uher et al., Contextualised behavioural measurements of personality differences obtained in behavioural tests and social observations in adult capuchin monkeys (Cebus apella), unpublished). In fact, it is well known in psychology that the cross-situational consistency of individual behaviour is often only moderate (Mischel 1968). Even within a given situation, the internal consistency of behaviours that are assigned to the same construct in both everyday and scientific psychology is often low to zero, such as with regard to gaze aversion, long pauses in speech, hesitant speaking, and restricted gestures all of which are assigned to the construct of "shyness" (Asendorpf 1988).

In a nutshell, it must be considered that behavioural data have different properties than assessment data because they capture different kinds of phenomena. The distributional patterns of behavioural events may differ from those of the mental representations that people develop of them. Behavioural measurements may not fulfil the psychometric standards that have been established for assessments that use predefined scales. This need not indicate insufficient utility for scientific investigations, but instead may reflect real patterns that are important and should be researched (Fenson et al. 2000). The normal curve distributions of individual scores that are frequently assumed for the five major dimensions (Jang et al. 2002; Loehlin et al. 1998) might simply result from processes of mental information processing, from the limited response formats of questionnaires, and from the artificial selection of only those questionnaire items that yield such distributional patterns in the target population (Uher et al., Contextualised behavioural measurements of personality differences obtained in behavioural tests and social observations in adult capuchin monkeys (Cebus apella), unpublished).

To meet these challenges, new meta-theoretical principles of data reduction have been proposed within the framework of the Behavioural Repertoire $\mathrm{x}$ Environmental Situations Approach (Uher, Methodological approaches to personality 
taxonomies: The Behavioural Repertoire x Environmental Situations ApproachA non-lexical alternative, unpublished; Uher et al., Contextualised behavioural measurements of personality differences obtained in behavioural tests and social observations in adult capuchin monkeys (Cebus apella), unpublished). They allow researchers to employ a two-step reduction procedure. First, behavioural data are reduced based on the particular behaviours' scientifically established functional importance - regardless of potentially low internal consistencies among behavioural measurements. Then the thus-derived composite measures of functionally defined constructs (rather than the raw behavioural data) are subjected to statistical reduction techniques. The first reduction step corresponds to the intuitive processes of mental (re)construction, but is, in contrast to them, made explicit and based on scientific knowledge. This step can therefore always be traced and reconsidered if needed.

Statistical principles of data analyses should also be reconsidered with regard to within-individual variability. Prevailing methods are based on the metatheoretical assumption that variability reflects variance derived from measurement error and from random variation around a hypothetical true score (i.e., a "trait"). But variability can be an important phenomenon in itself. Dynamic system theories, for example, consider variability to be a driving force of development and a potential indicator of ongoing processes. Studying variation patterns can therefore offer important insights into how individuals change and develop (Thelen and Smith 1993). Analytical principles that are adapted to the developmental nature of behavioural phenomena - and thus of personality - and that allow for analyses of variability should be further developed (see van Geert and van Dijk 2002).

Finally, interpretations should carefully consider what phenomena the obtained data (can) actually reflect (Gillespie and Zittaun 2010; Køppe 2012; Toomela 2011; Valsiner 2012). For example, analyses of lay people's assessments cannot reveal the relative contributions of genetic inheritance of and environmental influence on individual differences nor can they be insightful about their evolutionary genetics because assessments capture everyday psychological knowledge structures. This knowledge is transmitted nongenetically through behavioural and symbolic inheritance systems (Jablonka and Lamb 2005). The results reported in pertinent assessment studies can thus mean two things. If - despite fragmentary human perception, imperfect mental (re)construction, and socioculturally negotiated ascriptions of meaning - this knowledge accurately captures some real structures in the phenomena about which it has been developed, then they are promising. But if - through mental and social (re)construction-this knowledge reflects more structure than there actually is in the phenomena that are being constructed or if it reflects structures in phenomena other than the phenomena that this knowledge is assumed to be about, then these results may be misleading. Psychologists can find this out only if they study the phenomena that can be influenced directly and indirectly by genetic transmission (e.g., physiology, behaviour) rather than the pertinent symbols and representations that lay people develop of them.

f) Scientific language. A scientific language that transcends common sense and that encodes knowledge in specially established sign systems should be developed 
purposefully for personality psychology as has already been done for the (hard) natural sciences (cf. Valsiner 2012). For example, although the statistical derivation of taxonomic personality constructs is described explicitly and precisely in lexical research (e.g., with factor loadings), their meanings remain implicit because they are derived from abstract everyday psychological terms. These terms cannot be easily traced to concrete perceivable phenomena anymore and are loaded with implicit meanings that are specific to particular sociocultural communities. Instead, terminology for personality constructs that begins with specific terms and constructs that are close to the directly perceivable qualitative and quantitative properties of the defined study phenomena for encoding the data should be developed (cf. Desideratum 1d). Then, these specific constructs should be processed during the reduction and analysis of the data using formal-logical operations to generate in a stepwise fashion more abstract and complex constructs that can be clearly traced to the concrete references from which they were derived. For these traceable constructs, abstract, complex, and even new terms should be developed for scientific psychology. This will help to reduce the unintended confusion with everyday psychological terms that all personality researchers have in their minds as well. For example, thus-derived complex constructs of individual-specific patterns of behaviour can be labelled based on the bio-psycho-socio-ecological functions of the particular behaviours and environmental situations to which they refer (Uher 2008b; Uher et al., Contextualised behavioural measurements of personality differences obtained in behavioural tests and social observations in adult capuchin monkeys (Cebus apella), unpublished).

g) Structures that allow for the constant scrutinising of epistemology, ontology, and specific theories that have been developed about the phenomena studied. Metatheoretical (Toomela 2011), cultural and ethical structures that facilitate discussions at the philosophy-of-science level and that help to delimit the paradigmatic and ideological practices that hinder intellectual innovation should be established in psychology communities and their publication media. Among all seven issues, this may be the most difficult to achieve.

\section{Desideratum 2: Comprehensive Taxonomies of Individual-Specific Patterns in Behaviour and of Individual-Specific Patterns in Outer Appearance}

The structures of individual-specific patterns in behaviour and in outer appearance are still largely unknown (cf. Uher 2008a, b; Westen 1996). They should be researched and taxonomised using the philosophy-of-science framework outlined above (Desideratum 1) to the same systematic and comprehensive extent as has already been done for the knowledge structures of some language communities - for humans and for some other species as well. Three issues must be considered.

a) Behaviour is a phenomenon of the present and thus requires realtime recording. To capture the defined elements of behaviour in the flow of events, methods that help reduce the limitations that are entailed by human perceptual abilities and by the actions required for recording what is perceived should be used and further developed. For example, computerised life observations, video techniques, and software-assisted coding techniques enable the creation of detailed records of 
complex and quickly occurring events (e.g., Uher et al. 2008; Uher et al., Contextualised behavioural measurements of personality differences obtained in behavioural tests and social observations in adult capuchin monkeys (Cebus apella), unpublished). Methods of first-person recording (Lahlou 2011a, b) and of ambulatory monitoring (Fahrenberg and Myrtek 2001; Fahrenberg et al. 2007) enable computerised measurements of ongoing behaviours in daily life settings. Studies on outer appearances could employ photographical techniques.

b) Individual-specific patterns are temporal phenomena and are thus not phenomena of the present. Their identification requires repeated measurement occasions over time and posterior statistical analyses; they cannot be measured directly (Uher, Meta-theoretical foundations of objectivity versus subjectivity in quantifications of behaviour and personality, unpublished).

c) Phenomena of behaviour and of artificially modified outer appearance must be studied in the contexts in which they emerge. As phenomena that are functionally mediated by the present environment (Uher, What is behaviour? And (when) is language behaviour? A meta-theoretical definition, unpublished), behaviour and artificially modified outer appearance must be studied together with the contexts in which they emerge, such as particular situations, groups, populations (e.g., nations, sociocultural, or semiotic communities), times (e.g., generations, historical eras), and species. This requires taxonomic approaches for categorising contexts. Such can be taken and further developed from approaches that are designed to study the behavioural contexts of average individuals, such as from behaviour setting theory (Barker 1968; Schoggen 1989), world installation theory (Lahlou 2008; 2011a), cross-cultural psychology (Berry et al. 2011), and behavioural ecology. This also includes analyses of the similarities and differences of the obtained taxonomic structures of individual-specific behaviours and outer appearances within and across contexts to identify patterns that are specific to particular contexts or universal across multiple defined contexts (e.g., situations, sociocultural and semiotic communities, species; Uher 2008a, b, An integrative meta-theoretical framework for research on individual behaviour in context—situations, populations, species, unpublished).

Desideratum 3: Analyses of the Relations Between the Taxonomic Structures of Individual-Specific Behaviours and those of Individual-Specific Outer Appearances

The identified taxonomic structures of individual-specific patterns in behaviour and in outer appearances should then be analysed purposefully and systematically for interrelations between them. The background of defined contexts in which they have been studied (e.g., situations, groups, populations, times, species) must thereby be carefully considered.

\section{Desideratum 4: Analyses of How People Perceive and Construct Structures} of Individual-Specific Patterns of Behaviour and of Outer Appearance

Still little is known about what exactly people perceive in individual behaviour and in outer appearance in everyday life, and about what specific communities actually consider salient and relevant. "Although [the lexical hypothesis] is reasonably clear, the criteria of importance that have shaped the personality lexicons of everyday 
people are not well understood" (John et al. 1988, p. 175). For example, withinindividual variance often considerably exceeds between-individual variance in humans (Shweder and Sullivan 1990) and also nonhuman species (Uher, An integrative meta-theoretical framework for research on individual behaviour in contextsituations, populations, species, unpublished). But still, between-individual variability seems to be more salient to human minds and more central to pertinent representations. What actually happens during the processes of the perception and mental (re)construction of the perceived phenomena that this is possible? The concept of time-relative probabilities allows researchers to determine the magnitudes of withinindividual and between-individual variation (measured in set-theoretically objective ways, cf. Desideratum 1d) that particular people - both individuals and communities - perceive as salient in other humans and in some other species (Uher et al., Through the human personality glasses: Constructed personality taxonomy and typology in Crab-eating macaques (Macaca fascicularis), their crossmethod coherences and 24month stabilities, unpublished). Researchers should also study how people construct, interpret, appraise, explain, and represent the perceived (see e.g., Laucken 1974), and in which ways this may be different in different contexts (e.g., cultures and semiotic systems). In a nutshell, the processes of perception and mental and social construction that create such knowledge and their lexical encodings - rather than just their outcomes - should be systematically studied (Gillespie and Zittaun 2010; Jovchelovitch 2007; Komatsu 2012; Pillai 2012; Rosenbaum and Valsiner 2011; Westen 1996). A portfolio of methods (see Desideratum 1d) should thereby be used, qualitative and microgenetic ones in particular (Wagoner 2009; Valsiner 1998).

Desideratum 5: Comprehensive Taxonomies of Everyday Psychological Knowledge About Individual-Specific Patterns of Behaviour and of Outer Appearance

The pertinent bodies of everyday psychological knowledge have so far been taxonomised primarily by beginning with the systems of their lexical encodings (John et al. 1988). An entirely alternative strategy that begins with the phenomena that are represented in this knowledge (i.e., from behaviours and outer appearances) should be pursued. To taxonomise behaviour-related everyday psychological knowledge, the Behavioural Repertoire x Environmental Situations Approach (cf. Desideratum 1a) can be applied. For such investigations, the elements selected from the scientifically described behavioural ecology of a population or species are described in the everyday language of the target community. It is thereby important to use specific terms and descriptions that are close to perceivable qualitative properties (e.g., in contextualised behaviour-descriptive verb sentences). These descriptions can then be used in empirical investigations of pertinent representations using assessment methods or qualitative methods (see Desideratum 1d). This approach has already been applied to study (using assessments) the representations that human observers develop about individual-specific behaviours in other species; specifically those that zoo keepers developed about great ape individuals (Uher and Asendorpf 2008) and those that students and researchers developed about individual crab-eating macaques (Uher et al., Through the human personality glasses: Constructed personality taxonomy and typology in Crab-eating macaques (Macaca fascicularis), their crossmethod coherences and 24-month stabilities, unpublished). Similar approaches should be used to 
taxonomise everyday psychological knowledge about individual-specific outer appearances. Ideally, taxonomising knowledge structures should be based on the empirically established comprehensive taxonomies of individual-specific behaviours and outer appearances and on the knowledge about their empirical interrelations (see Desiderata 2 and 3). But because these are difficult to establish given the enormous logistic efforts required, the aforementioned approach will provide a viable alternative until such taxonomies can be established empirically.

In addition to descriptions, researchers should systematically study patterns of interpretation, appraisal, and explanation and compare them among sociocultural and language communities. Such analyses can be done only with qualitative methods, e.g., using interviews or analyses of texts, conversations, and reports (Lahlou 2011a). For example, Laucken (1974) systematically collected and catalogued all verbal manifestations of lay psychology explanations and predictions of human behaviour, such as in newspapers and everyday conversations that he came across over a period of 1.5 years. Then he analysed and categorised the structures and the conceptual elements underlying these verbal manifestations to reconstruct the "naïve behaviour theory" that people were using in everyday life in the population that he studied at the time of his investigation.

Desideratum 6: Analyses of the Relations Between the Taxonomic Structures of Everyday Psychological Knowledge and Those of Individual-Specific Behaviours and of Individual-Specific Outer Appearances

The taxonomies of everyday psychological knowledge should be set systematically in relation to the taxonomised structures of behaviours and outer appearances to explore convergences and divergences therein both within and between different cultural and semiotic communities. Such analyses will unravel how taxonomies about everyday psychological knowledge and those derived from their lexical encodings actually represent individual-specific patterns in behaviour and in outer appearance; the ways in which they may reflect different perceptions, interpretations, appraisals, and explanations; and how this may vary within and among different sociocultural and language communities. The above-mentioned studies on the representations of human observers of great apes and crab-eating macaques found systematic coherence between the human observers' pertinent represented knowledge and contextualised measurements of individual-specific behaviours (likewise selected using the Behavioural Repertoire x Environmental Situations Approach). But they also found interesting deviations that provide insight into how humans may perceive and (re)construct such patterns in these species (Uher 2011b).

\section{Desideratum 7: Systematic Investigations of Causally Related Phenomena}

The established descriptive-structural taxonomies of individual-specific behaviours and of outer appearances and the taxonomies of the related contexts (cf. Desideratum 2c) should be used to guide causal-explanatory investigations of these phenomena. This strategy has crucial advantages over approaches that begin with the causally related phenomena themselves because behaviours, outer appearances, and environments are exterospectively accessible and can therefore be systematically categorised and quantified in set-theoretically objective ways (see above). But this is not possible 
for psychological processes and for representations. Because they are not directly perceivable; approaches that begin with these phenomena are prone to conceptual biases on the part of the researchers. This is also in line with the methodological insight made by social-cognitive personality theorists that descriptive-structural investigations of behaviours in their situational contexts are essential for uncovering the psychological processes through which distinctive individual behaviours emerge and endure in specific situations (e.g., Wright and Mischel 1987; Wright and Zakriski 2003).

The phenomena that may be causally related to individual-specific behaviours and outer appearances, and to their pertinent representations should be conceptually integrated into the philosophy-of-science framework outlined above (for a metatheoretical definition of the psyche, see, e.g., Toomela 2010b). As with behavioural and psychological phenomena, it is important to study the potential causes of behaviours, outer appearances, and representations separately because they need not be the same for these different kinds of phenomena (cf. Toomela 2011). For example, the cognitive and social processes that create encoded knowledge structures (Witkowski and Brown 1978) can but do not necessarily causally influence behaviours and outer appearances as well. Given their order of emergence in ontogeny and phylogeny, they may not even be the primary causal influences of behaviour. Research on nonhuman species can be particularly insightful in this regard. Among causal phenomena internal to the individual, phenomena of physiology, (neuro)anatomy, and genes can be measured quantitatively because these are physical phenomena. But for investigations of psychological phenomena, three important issues must be considered.

a) Most psychological processes are unconscious (Freud 1915). Individuals are consciously aware of only small subsets of their psychological processes (Westen 1996, 1999) and many of these processes cannot be easily verbalised (Brower 1949; Kelly 1955; Komatsu 2012; Valsiner 2012).

b) Psychological phenomena are entirely internal and accessible only through introspection. Psychological events cannot be perceived in other individuals (Locke 1689; Toomela 2008, 2011). They can only be inferred from phenomena that are external and that may mediate between an individual's psychological events and his or her environment (see above, Fig. 1), such as behavioural phenomena and some parts of human language (Uher, What is behaviour? And (when) is language behaviour? A meta-theoretical definition, unpublished). In oneself, psychological events can be directly perceived - through introspection. But scientific introspection imposes challenges because reflection and attention inevitably introduce changes in the course of psychological events (Wundt 1904). Moreover, the results of introspection can be investigated only based on their externalisation through behaviour and language. Direct investigations of psychological phenomena are impossible.

c) Psychological phenomena are bound to the present and thus can be studied only in the present (Gillespie and Zittaun 2010; Uher, What is behaviour? And (when) is language behaviour? A meta-theoretical definition, unpublished; Valsiner 1998, 2012). Disentangling constructs about psychological phenomena (e.g., anxiousness) from ongoing events of psychological phenomena (e.g., experiencing anxiety 
in a given moment) is difficult because both are internal and are externalised primarily in a verbal manner. For the phenomena of thought, this entails particular difficulties because constructs are the memorised resultants of past thoughts (Peirce 1902, CP 2.84; Valsiner 2012). The lexical encodings used to externalise psychological events are constructs as well (Vygotsky 1962), including those that refer to ongoing events of psychological phenomena. Given that one can never ascertain whether words denoting particular psychological phenomena actually refer to exactly the same phenomena in different individuals (Locke 1689, see above), (re)constructions by investigators are inevitably involved in research on psychological phenomena.

The fact that all psychological phenomena are actualities and inherently bound to the present (Gillespie and Zittaun 2010; Valsiner 1998, 2012) opens up some ways - within the limitations mentioned - in which ongoing psychological events can be distinguished from pertinent constructs because constructs inherently also involve events from the past. As for behavioural phenomena, this requires realtime investigations of ongoing psychological phenomena, such as through microgenetic methods (Wagoner 2009) or methods of experience sampling in real-life settings (Mehl and Connor 2012). But because psychological phenomena cannot be investigated directly, this requires that researchers differentiate their qualitative properties by explicitly categorising the phenomena through which they can be externalised (see Desideratum 1d). These categories may refer, for example, to particular information externalised in language (e.g., complains about pains, expressed worries) or to nonverbal behaviours. Humans usually make these assignments intuitively, for example, between behavioural and psychological assignments as is apparent in concepts of "emotional behaviour" or "helping" that already contain assumptions about underlying emotions or intentions. Because these assumptions may be erroneous, in particular if individual members of other sociocultural communities and of other species are concerned, the assignment of externalised phenomena to particular psychological phenomena should be made explicit to enable continuous scrutinising. For quantifications of individual-specific patterns, realtime records of events of externalisation categories should be repeatedly obtained and accumulated over time. Probabilistic, differential, and temporal patterns of their occurrences can then be quantified based on time-relative probabilities (see above). Compared to the ease of obtaining lay people's assessments, this may appear to be a fairly cumbersome and rough way to investigate psychological phenomena. But given their peculiarities, this may be the only way in which the psychological phenomena themselves - rather than lay people's knowledge about them - can be somewhat quantified in set-theoretically objective ways.

\section{Desideratum 8: Investigations of Ontogenetic Development}

The development of individual-specific behaviours, outer appearances, pertinent knowledge structures, and encodings should be studied systematically. Close attention should be paid to the fact that, in present-day humans, none of these patterns can be explored and understood independently of the others because they always develop in tight dialectic interplay. Methods of investigation and developmental theories must 
therefore be primarily dialectical rather than demonstrative as those currently prevailing in psychology (Rychlak 1968; Sameroff 2010; Valsiner 2012). Particularly illuminative are investigations of early ontogeny when the patterns of behaviour and outer appearances of human individuals can be studied before and while these individuals develop the abilities to mentally construct pertinent representations and acquire complex repertoires of socioculturally shared meanings and symbols. The influences of the latter should be explored in comparative investigations of a broad range of human cultures (Keller 2007) by explicitly considering cultures that are markedly different from the Western societies on which much of today's personality psychology is focused. Adult individuals should be studied with regard to their own behaviours, outer appearances, and pertinent representations, but also with regard to the representations that they develop about young children who do not yet share the adults' social representations comprehensively and whose behaviours are therefore still not so strongly influenced by the sociocultural appraisals they comprise.

Research on various nonhuman species will illuminate patterns of ontogenetic development of individual-specific behaviours (Suomi 2005) and also of individualspecific outer appearances that may be universal to some groups of species. For example, in various species such as horses and dogs, individuals with more gracile bones and limbs also seem to differ in their individual-specific behaviours from conspecifics that are more robust in their physical appearance (Grandin and Johnson 2005). Furthermore, the abilities that are prerequisite for the recognition and mental reconstruction of individual-specific patterns in behaviour should be studied in various species as well as in humans with special abilities (e.g., eidetic perception) or particular impairments (e.g., deficiencies in time perception). Differences in these abilities should be set in relation to evidence that may indicate the recognition and reconstruction of individual-specific patterns, such as individualised dyadic relationships. Research on nonhuman individuals that have acquired semiotic systems that are socially shared with humans can provide insights into the role that language may have played in the development of pertinent abilities during human evolution.

\section{Desideratum 9: Alternative Conclusions from the Lexical Hypothesis}

So far, psychologists have deduced just one conclusion from the lexical hypothesis (i.e., all pertinent terms in the lexica constitute the universe of elements for empirical studies). Alternative deductive conclusions should be derived systematically, such that person perceptions may also be encoded in idioms and proverbs. In contrast to single words, idioms and proverbs usually do not translate well; literal translations often change their meaning or render them meaningless. What do these different ways of encoding knowledge reveal about the different sociocultural and linguistic communities and their ways of representing their social world?

Furthermore, inductive conclusions should be derived and facts should be sought that may support or even falsify the hypothesis. For example, researchers should purposefully study how people construct and encode the phenomena that they perceive, even when these perceptions are not conscious (e.g., subliminal odours, pheromones). The fact that within-individual differences are not mentioned in the 
lexical hypothesis should be scrutinised. Do people perceive and mentally represent this at all? And do they notice that some individuals are more consistent within themselves and across situations, whereas others are not both in humans (cf. Caspi and Roberts 1999; Mischel et al. 2002) and in (at least some) nonhuman species as well (Uher et al., Contextualised behavioural measurements of personality differences obtained in behavioural tests and social observations in adult capuchin monkeys (Cebus apella), unpublished)?

\section{Desideratum 10: Alternative Nonlexical Hypotheses}

Fundamentally new hypotheses about individual-specificity should be developed, in particular about the theoretical nature of the phenomena in which it can be found, why it emerges in these phenomena at all, and how. These questions can be explored only if personality psychology dismisses its narrow research focus on Homo sapiens and the phenomena reflected in its language.

The shift in perspective from humans to the enormous diversity of today's species opens a huge field of research that allows profound and illuminative insights .... What is unique about Homo sapiens compared to all other species in the phylogenetic tree? What personality [differences ${ }^{4}$ ] may have contributed to Homo sapiens' accelerated development in the most recent evolutionary past and to its unmatched success in conquering almost every habitat on earth? There is no better opportunity to understand the phylogenetic basis, adaptive significance and ecological relevance of personality and its role in speciation than studying the evolved diversity of species. (Uher 2008a, p. 427-428)

The much wider focus of cross-species comparative psychology may challenge some deep-rooted ideologies about human personality. But it opens doors to alternative hypotheses that trigger new approaches and insights into the phylogenetic history of these fascinating phenomena in humans and other species, such as the personality constructs-promote-peaceful-anonymous-contacts hypothesis, the language-promotes-individualisation hypothesis (see above), the central nervous system hypothesis, or the motility hypothesis (Uher 2009) to name just a few. All these new hypotheses - just as the lexical hypothesis is - are centred on the fascinating finding that individual members of the human species as well as of other species develop individual-specific styles of interaction with their environments that we construct as personality.

Those who say it can’t be done are usually interrupted by others doing it. (James Arthur Baldwin)

\footnotetext{
${ }^{4}$ The attentive reader will notice that the article referred to here and some additional ones are written based on the assumptions of trait psychology. This documents the educational origins of the author and the metatheoretical development of her work.
} 
Acknowledgments I am very grateful to Manfred Schmitt, Jochen Fahrenberg, Jaan Valsiner, Grete Arro, and an anonymous reviewer for thoughtful comments on earlier drafts of the manuscript. The views expressed herein are mine and should not be attributed to any of the persons who provided commentaries. Preparation of the manuscript was supported by a grant from the Deutsche Forschungsgemeinschaft DFG (grant number UH249/1-1).

Open Access This article is distributed under the terms of the Creative Commons Attribution License which permits any use, distribution, and reproduction in any medium, provided the original author(s) and the source are credited.

\section{References}

Ackerman, P. L. (1994). Intelligence, attention, and learning: Maximal and typical performance. In D. K. Detterman (Ed.), Current topics in human intelligence: Volume 4. Theories of intelligence (pp. 1-27). Norwood: Ablex.

Allport, G. W. (1937). Personality: A psychological interpretation. New York: Macmillan.

Allport, G. W. (1961). Pattern and growth in personality. New York: Holt, Rinehart and Winston.

Allport, G. W., \& Odbert, H. G. (1936). Trait names: a psycholexial study. Psychological Monographs, 47, 1.

Althusser, L., \& Balibar, E. (1970). Reading capital. London: New Left Books.

Angleitner, A., Ostendorf, F., \& John, O. P. (1990). Towards a taxonomy of personality descriptors in German: a psycho-lexical study. European Journal of Personality, 4, 89-118.

Aristotle (1963[1936]). Physiognomics. In Aristotle. Minor works (pp. 81-137). trans. W. S. Hett. Cambridge, MA and London: Harvard University Press.

Arro, G. (2012). Peeking into personality test answers: Inter- and intraindividual variety in item interpretations. Integrative Psychological and Behavioral Science. doi:10.1007/s12124-012-9216-9.

Asendorpf, J. B. (1988). Individual response profiles in the behavioral assessment of personality. European Journal of Personality, 2, 155-167.

Ashton, M. C., \& Lee, K. (2005). A defence of the lexical approach to the study of personality structure. European Journal of Personality, 19, 5-24.

Augustine, Saint (397-398 AD/1961). Confessions. Translated by Pinecoffin, R. S. (Ed.), Harmondsworth: Penguin.

Bandura, A. (1986). Social foundations of thought and action. Englewood Cliffs: Prentice Hall.

Barker, R. G. (1968). Ecological psychology. Concepts and methods for studying the environment of human behavior. Stanford University Press

Baumeister, R. F., Vohs, K. D., \& Funder, D. C. (2007). Psychology as the science of self-reports and finger movements: whatever happened to actual behavior? Perspectives on Psychological Science, 2, 396-403.

Belyaev, D. K. (1969). Domestication of animals. Science Journal (U.K.), 5, 47-52.

Berry, J. W., Poortinga, Y. H., Breugelmans, S. M., Chasiotis, A., \& Sam, D. (2011). Cross-cultural psychology. Research and applications. Cambridge: Cambridge University Press.

Bhutta, M. (2007). Sex and the nose: human pheromonal responses. Journal of the Royal Society of Medicine, 100, 268-274.

Biesanz, J. C., \& Human, L. J. (2010). The cost of forming more accurate impressions: accuracy motivated perceivers see the personality of others more distinctively but less normatively. Psychological Science, 24, 589-594.

Blaffer-Hrdy, S. (2009). Mothers and others: the evolutionary origins of mutual understanding. Cambridge: Belknap.

Block, J. (1995). A contrarian view of the five-factor approach to personality description. Psychological Bulletin, 117, 187-229.

Block, J. (2001). Millenial contrarianism: the five factor approach to personality description 5 years later. Journal of Research in Personality, 35, 98-107.

Block, J. (2010). The five-factor framing of personality and beyond: some ruminations. Psychological Inquiry, 21, 2-25.

Bock, P. K. (2000). Culture and personality revisited. American Behavioural Scientist, 44, 32-40.

Bolden, R., \& Moscarola, J. (2000). Bridging the quantitative-qualitative divide: the lexical approach to textual data analysis. Social Science Computer Review, 18, 450-460.

Bordt, M. (1998). Platon Werke. Übersetzung und Kommentar, Band V 4. Lysis. Göttingen: Vandenhoeck \& Ruprecht. 
Borkenau, P., \& Liebler, A. (1992). Trait inferences: sources of validity at zero acquaintance. Journal of Personality and Social Psychology, 62, 645-657.

Borkenau, P., \& Ostendorf, F. (2008). NEO-Fünf-Faktoren-Inventar nach Costa \& McCrae. 2. neu normierte und vollständig überarbeitete Auflage. Göttingen: Hogrefe.

Borkenau, P., Mauer, N., Riemann, R., Spinath, F. M., \& Angleitner, A. (2004). Thin slices of behavior as cues of personality and intelligence. Journal of Personality and Social Psychology, 86, 599-614.

Bouts, P. (1931/1986). La psychognomie. Paris: Dervy-Livres.

Brody, N. (1994). Heritability of traits. Psychological Inquiry, 5, 117-119.

Bronfenbrenner, U. (1979). The ecology of human development: Experiments by nature and design. Cambridge: Harvard University Press.

Brower, D. (1949). The problem of quantification of psychological science. Psychological Review, 56, 325-331.

Brown, D. E. (1991). Human universals. New York: McGraw-Hill.

Brunswik, E. (1952). The conceptual framework of psychology. Chicago: The University of Chicago Press.

Brunswik, E. (1955). Representative design and probabilistic theory in a functional psychology. Psychological Review, 62, 193-217.

Carrasco, M. C., Bernal, M. C., \& Redolat, R. (2001). Time estimation and aging: a comparison between young and elderly adults. International Journal of Aging \& Human Development, 52, 91-101.

Caspi, A., \& Roberts, B. W. (1999). Personality continuity and change across the life course. In Pervin, L. A. \& John O. P. (Eds). Handbook of Personality: Theory and Research (pp. 300-326, $2^{\text {nd }}$ ed). New York, NY: Guilford Press.

Caspi, A., \& Roberts, B. W. (2001). Personality development across the life course: the argument for change and continuity. Psychological Inquiry, 12, 49-66.

Cattell, R. B. (1943). The description of personality I. Foundations of trait measurement. Psychological Review, 50, 559-594.

Cattell, R. B. (1950). An introduction to personality study. London: Hutchinson.

Cattell, R. B. (1952). Factor analysis. New York: Wiley.

Cervone, D., Shadel, W. G., \& Jencius, S. (2001). Social-cognitive theory of personality assessment. Personality and Social Psychology Review, 5, 33-51.

Church, A. T., \& Katigbak, M. S. (1988). The emic strategy in the identification and assessment of personality dimensions in a non-Western culture: Rationale, steps, and a Philippine illustration. Journal of Cross-Cultural Psychology, 19, 140-163.

Church, A. T., \& Katigbak, M. S. (1989). Internal, external, and self-report structure of personality: An investigation of cross-language and cross-cultural generalizability. Journal of Personality and Social Psychology, 57, 857-872.

Conley, J. J. (1984). Longitudinal consistency of adult personality: self-reported psychological characteristics across 45 years. Journal of Personality and Social Psychology, 47, 1325-1333.

Costa, P. T., Jr., \& McCrae, R. (1992). Four ways five factors are basic. Personality and Individual Differences, 13, 653-665.

Darwin, C. R. (1859). On the origin of species by means of natural selection, or the preservation of favoured races in the struggle for life. London: John Murray.

Daston, L., \& Galison, P. (2007). Objectivity. New York: Zone Books.

De Raad, B. (1998). Five big, Big Five issues: Rationale, content, structure, status, and crosscultural assessment. European Psychologist, 3, 113-124.

De Weerth, C., van Geert, P., \& Hoitink, H. (1999). Intra-individual variability in infant behavior. Developmental Psychology, 35, 1102-1112.

Digman, J. M. (1990). Personality structure: emergence of the five-factor model. Annual Review of Psychology, 41, 417-440.

Diriwächter, R., Valsiner, J. \& Sauck, C. (2004). Microgenesis in Making Sense of Oneself: Constructive Recycling of Personality Inventory Items. Forum Qualitative Sozialforschung/Forum: Qualitative Social Research, 6, Art. 11.

Dunbar, R. (1996). Grooming, gossip, and the evolution of language. Cambridge: Harvard University Press.

Eysenck, H. J. (1947). Dimensions of personality. London: Routledge and Kegan Paul.

Eysenck, H. J. (1992). Four ways five factors are not basic. Personality and Individual Differences, 13, $667-673$.

Fahrenberg, J., \& Myrtek, M. (Eds.). (2001). Progress in ambulatory assessment. Computerassisted psychological and psychophysiological methods in monitoring and field studies. Göttingen: Hogrefe \& Huber.

Fahrenberg, J., Myrtek, M., Pawlik, K., \& Perrez, M. (2007). Ambulatory assessment-monitoring behavior in daily life settings. A behavioral-scientific challenge for psychology. European Journal of Personality Assessment, 23, 206-213. 
Fenson, L., Bates, E., Dale, P., Goodman, J., Reznick, J. S., \& Thal, D. (2000). Measuring variability in early child language: Don't shoot the messenger. Comment on Feldman et al. Child Development, 71, $323-328$.

Fraisse, P. (1964). The psychology of time. London: Eyre \& Spottiswoode.

Freud, S. (1915). The unconscious. Standard Edition, 14, 159-214.

Funder, D. C. (2004). The personality puzzle (3rd ed.). New York: W.W. Norton \& Co.

Galton, F. (1884). Measurement of character. Fortnightly Review, 36, 179-185.

Germonpré, M., Sablin, M. V., Stevens, R. E., Hedges, R. E. M., Hofreiter, M., Stiller, M., et al. (2009). Fossil dogs and wolves from Palaeolithic sites in Belgium, the Ukraine and Russia: Osteometry, ancient DNA and stable isotopes. Journal of Archaeological Science, 36, 473-490.

Gigerenzer, G. (1991). From tools to theories: a heuristic of discovery in cognitive psychology. Psychological Review, 98, 254-267.

Gigerenzer, G., Todd, P., \& the ABC Group. (1999). Simple heuristics that make us smart. New York: Oxford University Press.

Gillath, O., Bahns, A. J., Ge, F., \& Crandall, C. S. (online 2012). Shoes as a source of first impressions. Journal of Research in Personality.

Gillespie, A., \& Zittaun, T. (2010). Studying the moment of thought. In A. Toomela \& J. Valsiner (Eds.), Methodological thinking in psychology: 60 years gone astray? (pp. 69-88). Charlotte: Information age publishing.

Gillies, D. (2000). Varieties of propensity. The British Journal for the Philosophy of Science, 51, 807-835.

Goethe, J. W. (1907). In M. Hecker (Ed.), Maximen und reflexionen. Schriften der goethe-gesellschaft, 21. Weimar: Verlag der Goethe-Gesellschaft.

Goldberg, L. R. (1981). Language and individual differences: The search for universals in personality lexicons. In L. Wheeler (Ed.), Review of personality and social psychology: Vol. 2 (pp. 141-165). Beverly Hills: Sage.

Goldberg, L. R. (1982). From Ace to zombie: Some explorations in the language of personality. In C. D. Spielberger \& J. N. Butcher (Eds.), Advances in personality assessment (Vol. 1) (pp. 203-234). Hillsdale: Erlbaum.

Goldberg, L. R. (1990). An alternative "description of personality": the big-five factor structure. Journal of Personality and Social Psychology, 59, 1216-1229.

Goldberg, L. R. (1993). The structure of phenotypic personality traits. American Psychologist, 48, $26-34$.

Goodall, J. (1986). The chimpanzees of gombe: Patterns of behavior. Cambridge: The Belknap Press.

Grandin, T., \& Johnson, C. (2005). Animals in translation. New York: Scribner.

Grammer, K., Fink, B., \& Neave, N. (2005). Human pheromones and sexual attraction. European Journal of Obstetrics, Gynecology, and Reproductive Biology, 111, 135-142.

Gray, P. (1999). Psychology (3rd ed.). New York: Worth.

Guilford, J. P. (1959). Personality. New York: McGraw-Hill.

Hartung, F.-M., \& Renner, B. (2011). Social curiosity and interpersonal perception: a judge $\mathrm{x}$ trait interaction. Personality and Social Psychology Bulletin, 37, 796-814.

Hausheer, H. (1937). St. Augustine's conception of time. Philosophical Review, 46, 503-512.

Heine, S. J., Lehman, D. R., Peng, K., \& Greenholtz, J. (2002). What's Wrong with cross-cultural comparisons of subjective likert scales?: the reference-group effect. Journal of Personality and Social Psychology, 82, 903-918.

Heisenberg, W. (1989). Encounters with Einstein: And other essays on people, places, and particles. Princeton University Press.

Hempel, C. G., \& Oppenheim, P. (1948). Studies in the logic of explanation. Philosophy of science, 15, 135-75; reproduced in Hempel, Carl G. (1965). Aspects of scientific explanation. New York: Free Press.

Howard, G. S. (1994). Why do people say nasty things about self-reports? Journal of Organizational Behavior, 15, 399-404.

Howarth, C., Foster, J., \& Dorrer, N. (2004). Exploring the potential of the theory of social representations in community-based health research - and vice versa? Journal of Health Psychology, 9, 229-245.

Inoue S., \& Matsuzawa, T. (2007). Working memory of numerals in chimpanzees. Current Biology, 17, R1004-R1005.

Jablonka, E., \& Lamb, M. (2005). Evolution in four dimensions-Genetic, epigenetic, behavioral, and symbolic variation in the history of life. Cambridge: MIT Press.

James, W. (1893). The principles of psychology. New York: H. Holt and Company.

Jang, K. L., Livesley, W. J., Angleitner, A., Riemann, R., \& Vernon, P. A. (2002). Genetic and environmental influences on the covariance of facets defining the domains of the five factor model of personality. Personality and Individual Differences, 33, 83-101. 
John, O. P. (1990). The "Big Five" factor taxonomy: Dimensions of personality in the natural language and in questionnaires. In L. A. Pervin (Ed.), Handbook of personality: Theory and research (pp. 66-100). New York: Guilford.

John, O. P., \& Srivastava, S. (1999). The big five trait taxonomy: History, measurement, and theoretical perspectives. In L. A. Pervin \& O. P. John (Eds.), Handbook of personality: Theory and research (2nd ed., pp. 102-138). New York: Guilford.

John, O. P., Angleitner, A., \& Ostendorf, F. (1988). The lexical approach to personality: a historical. Review of trait taxonomic research. European Journal of Personality, 2, 171-203.

Joubert, C. E. (1984). Structured time and subjective acceleration of time. Perceptual and Motor Skills, 59, 335-336.

Jovchelovitch, S. (2007). Knowledge in context: Representations, community and culture. London: Routledge.

Kagan, J. (1994). Galen's prophecy. New York: Basic Books.

Kagan, J. (1998). Three seductive ideas. Cambridge: Harvard University Press.

Kagan, J., Reznick, J. S., \& Snidman, N. (1988). Biological bases of childhood shyness. Science, 240, 167-171.

Kaminski, J., Call, J., \& Fischer, J. (2004). Word learning in a domestic dog: evidence for 'fast mapping'. Science, 304, 1682-1683.

Keller, H. (2007). Cultures of infancy. New York: Tailor and Francis.

Kelly, G. A. (1955). The psychology of personal constructs (Vol. 1 And 2). New York: Norton.

Komatsu, K. (2012). Temporal reticence of the self: who can know my self? Integrative Psychological and Behavioral Science, 46, 357-372.

Køppe, S. (2012). A moderate eclecticism: ontological and epistemological issues. Integrative Psychological and Behavioral Science, 46, 1-19.

Kramer, R. S. S., \& Ward, R. (2010). Internal facial features are signals of personality and health. Quarterly Journal of Experimental Psychology, 63, 2273-2287.

Kretschmer, E. (1977). Körperbau und Charakter (1st ed. 1921) (26th ed.). Berlin: Springer.

Kuhn, T. S. (1962). The structure of scientific revolutions (1st ed.). Chicago: University of Chicago Press.

Kuhn, T. S. (1976). The function of dogma in scientific research. In I. R. Averill (Ed.), Patterns of psychological thought (pp. 47-66). Washington: Hemisphere Publishing Co.

Lahlou, S. (1996). Propagation of social representations. Journal for the Theory of Social Behaviour, 26, $157-175$.

Lahlou, S. (2001). Functional aspects of social representations. In K. Deaux \& G. Philogene (Eds.), Representations of the social: Bridging theoretical traditions (pp. 131-146). Oxford: Blackwell.

Lahlou, S. (2008). L'Installation du Monde: De la représentation à l'activité en situation. Aix-en-Provence, Université de Provence: Habilitation à Diriger des Recherches en Psychologie, 375.

Lahlou, S. (2011a). How can we capture the subject's perspective?: an evidence-based approach for the social scientist. Social Science Information, 50, 607-655.

Lahlou, S. (2011b). Socio-cognitive issues in human-centred design for the real world. In G. Boy (Ed.), The handbook of human-machine interaction (pp. 165-188). Aldershot: Ashgate.

Lamiell, J. T. (2003). Beyond individual and group differences: Human individuality, scientific psychology, and William Stern's critical personalism. Thousand Oaks: Sage Publications.

Laucken, U. (1974). Naive verhaltenstheorie. Stuttgart: Klett.

Laureys, S., Pellas, F., Van Eeckhout, P., Ghorbel, S., Schnakers, C., Perrin, F., et al. (2005) The locked-in syndrome: what is it like to be conscious but paralyzed and voiceless? Progress in Brain Research, $150,495-511$.

Lavater, J. C. (1775-1778). Physiognomische fragmente physiognomische fragmente, zur beförderung der menschenkenntniß und menschenliebe. (Bde. I-IV.). Leipzig und Winterthur: Weidmann und Steiner.

Lazarus, A. A. (1981). The practice of multimodal therapy: Systematic, comprehensive, effective psychotherapy. New York: McGraw-Hill.

Le Poidevin, R. (2004). A puzzle concerning time perception. Synthese, 142, 109-142.

Le Poidevin, R. (2011). The experience and perception of time. In E. N. Zalta (Ed.), The Stanford Encyclopedia of Philosophy (Fall 2011 Edition). http://plato.stanford.edu/archives/fall2011/entries/ time-experience/.

Lehner, P. N. (1998). Handbook of ethological methods. Cambridge: Cambridge University Press.

Lewin, K. (1935). A dynamic theory of personality. Selected papers. New York: McGraw-Hill.

Li, W., Moallem, I., Paller, K. A., \& Gottfried, J. A. (2007). Subliminal smells can guide social preferences. Psychological Science, 18, 1044-1049.

Locke, J. (1689/1975). Essay concerning human understanding. Book II. (chapter 32). Oxford: Oxford University Press. 
Loehlin, J. C., McCrae, R. R., Costa, P. T., Jr., \& John, O. P. (1998). Heritabilities of common and measurespecific components of the big five personality factors. Journal of Research in Personality, 32, 431453.

Loftus, G. R. (1996). Psychology will be a much better science when we change the way we analyze data. Current Directions in Psychological Science, 5, 161-171.

Ludlow, P., Nagasawa, Y., \& Stoljar, D. (Eds.) (2004). There's something about Mary. Essays on phenomenal consciousness and Frank Jackson's knowledge argument. MIT Press/A Bradford Book.

Marx, K. (1867). Das kapital. Buch I. Hamburg: Verlag Otto Meissner.

Matthews, G., Deary, I. J., \& Whiteman, M. C. (2003). Personality traits (2nd ed.). Cambridge: Cambridge University Press.

McAdams, D. P. (1985). Power, intimacy, and the life story: Personological inquiries into identity. New York: Guilford.

McAdams, D. P. (1992). The five-factor model in personality: a critical appraisal. Journal of Personality, 60, 329-361.

McAdams, D. P. (1994). A psychology of the stranger. Psychological Inquiry, 5, 145-148.

McCrae, R. (2009). The physics and chemistry of personality. Theory and Psychology, 19, 670-687.

McCrae, R. R., \& Costa, P. T., Jr. (1997). Personality trait structure as a human universal. American Psychologist, 52, 509-516.

McCrae, R. R., \& Costa, P. T., Jr. (2008). The five-factor theory of personality. In O. P. John, R. W. Robins, \& L. A. Pervin (Eds.), Handbook of personality: Theory and research (3rd ed., pp. 159-180). New York: Guilford.

Meehl, P. (1992). Factors and taxa, traits and types, differences of degree and differences of kind. Journal of Personality, 60, 117-174.

Mehl, M. R., \& Connor, T. S. (Eds.). (2012). Handbook of research methods for studying daily life. New York: Guilford Press.

Michell, J. (1997). Quantitative science and the definition of measurement in psychology. British Journal of Psychology, 88, 355-383.

Michell, J. (1999). Measurement in psychology. Cambridge: Cambridge University Press.

Michell, J. (2003). The quantitative imperative: positivism, naïve realism and the place in quantitative methods in psychology. Theory and Psychology, 13, 5-31.

Millikan, R. (1993). White queen psychology and other essays for Alice. Bradford: MIT Press.

Mischel, W. (1968). Personality and assessment. New York: Wiley.

Mischel, W., \& Shoda, Y. (1994). Personality psychology has two goals: must it be two fields? Psychological Inquiry, 5, 156-158.

Mischel, W., \& Shoda, Y. (1995). A cognitive-affective system theory of personality: reconceptualizing situations, dispositions, dynamics, and invariance in personality structure. Psychological Review, 102, 246-268.

Mischel, W., Shoda, Y., \& Mendoza-Denton, R. (2002). Situation-behavior profiles as a locus of consistency in personality. Current Directions in Psychological Science, 11, 50-54.

Molenaar, P. C. (2004a). A manifesto on psychology as idiographic science: bringing the person back into scientific psychology, this time forever. Measurement: Interdisciplinary Research and Perspectives, 2 , 201-218.

Molenaar, P. C. (2004b). Forum discussion of the manifesto's aggregational act. Measurement: Interdisciplinary Research and Perspectives, 2, 248-254.

Moscovici, S. (1961). La psychanalyse, son image et son public. Paris: PUF.

Moscovici, S. (1984). The phenomenon of social representations. In R. M. Farr \& S. Moscovici (Eds.), Social representations (pp. 3-70). Cambridge: Cambridge University Press.

Murray, H. A. (1938). Explorations in personality. New York: Oxford University Press.

Neuman, Y., Turney, P. D., \& Cohen, Y. (2012). How language enables abstraction: a study in computational cultural psychology. Integrative Psychological and Behavioral Science, 46, 129-145.

Norman, T. (1967). 2,800 personality trait descriptors: Normative operating characteristics for a university population. Ann Arbor: Department of Psychology, University of Michigan.

Omi, Y. (2012). Tension between the theoretical thinking and the empirical method: is it an inevitable fate for psychology? Integrative Psychological and Behavioral Science, 46, 118-127.

Patterson, F., \& Linden, E. (1981). The education of Koko. New York: Holt, Rinehart and Winston.

Paunonen, S. V., \& Ashton, M. C. (2001). Big five factors and facets and the prediction of behavior. Journal of Personality and Social Psychology, 81, 524-539.

Pawlik, K. (2006). Objektive tests in der persönlichkeitsforschung. In T. M. Ortner, R. T. Proyer, \& K. D. Kubinger (Eds.), Theorie und praxis objektiver persönlichkeitstests (pp. S. 16-S. 23). Bern: Hans Huber. 
Peirce, C. S. (1901/1935). Collected papers of Charles sanders Peirce (CP 7.218-1901, On the logic of drawing history from ancient documents especially from testimonies). Cambridge: Harvard University Press.

Peirce, C. S. (1902/1958). The simplest mathematics (CP 4.227-323). In Collected papers of Charles Sanders Peirce, Vols. 1-6, C. Hartshorne \& P. Weiss (eds.), vols. 7-8, A. W. Burks (ed.), Cambridge, MA: Harvard University Press. 1931-1935.

Peirce, C. S. (1903/1997). Lecture five: the normative sciences. In C. S. Peirce (Ed.), Pragmatism as a principle and method of right thinking (pp. 205-220). Edited by P. Turrisi. Albany, NY: Suny Press [Harvard Lectures on Pragmatism-CP 5.188-89].

Pepperberg, I. M. (2002). In search of King Solomon's ring: cognitive and communicative studies of grey parrots (Psittacus erithacus). Brain, Behavior and Evolution, 59, 54-67.

Pervin, L. A., \& John, O. P. (1997). Personality: Theory and research (7th ed.). New York: Wiley.

Piaget, J. (1969). The child's perception of time. London: Routledge \& Kagan Paul.

Pillai, P. (2012). Cultural directions and origins of everyday decisions. Integrative Psychological and Behavioral Science, 46, 235-242.

Popper, K. R. (1959). The propensity interpretation of probability. The British Journal for the Philosophy of Science, 37, 25-42.

Popper, K. R. (1934). Logik der forschung. Wien: Springer.

Rosenbaum, P. J., \& Valsiner, J. (2011). The un-making of a method: from rating scales to the study of psychological processes. Theory and Psychology, 21(1), 47-65.

Rosenthal, V. (2004). Microgenesis, immediate experience and visual processes in reading. In A. Carsetti (Ed.), Seeing, thinking and knowing-Meaning and self-organisation in visual cognition and thought, (pp. 221-243). Kluwer Academic Publishers.

Rotter, J. B. (1954). Social learning and clinical psychology. New York: Prentice-Hall.

Rychlak, J. F. (1968). A philosophy of science for personality theory. Boston: Houghton Mifflin.

Sameroff, A. (2010). A unified theory of development: a dialectic integration of nature and nurture. Child Development, 81, 6-22.

Sato, T., Wakabayashi, K., Nameda, A., Yasuda, Y., \& Watanabe, Y. (2010). Understanding a personality as a whole. In A. Toomela \& J. Valsiner (Eds.), Methodological thinking in psychology: 60 years gone astray? (pp. 89-119). Charlotte: InfoAge Publications.

Saucier, G., \& Goldberg, L. R. (1998). What is beyond the big five? Journal of Personality, 66, $495-524$.

Savage-Rumbaugh, E. S., \& Fields, W. M. (2000). Linguistic, cultural and cognitive capabilities of bonobos (Pan paniscus). Culture \& Psychology, 6, 131-153.

Schoggen, P. (1989). Behavior settings. A revision and extension of Roger G. Barker's ecological psychology. Stanford University Press.

Schwarz, N. (1999). Self-reports: how the questions shape the answer. American Psychologist, 54, 93-105.

Schwarz, M. (2009). Is psychology based on a methodological error? Integrative Psychological and Behavioral Science, 43, 185-213.

Sheldon, W. H. \& Stevens, S. S. (1942). The varieties of temperament: a psychology of constitutional differences. (1st ed). Harper \& Brother.

Shevlin, M., Walker, S., Davies, M. N. O., Banyard, P., \& Lewis, C. A. (2003). Can you judge a book by its cover? Evidence of self-stranger agreement on personality at zero acquaintance. Personality and Individual Differences, 35, 1373-1383.

Shweder, R. A., \& D’Andrade, R. G. (1980). The systematic distortion hypothesis. In R. A. Shweder (Ed.), Fallible judgement in behavioral research: New directions for the methodology in social and behavioral science, Vol. 4 (pp. 37-58). San Francisco: Jossey-Bass.

Shweder, R. A., \& Sullivan, M. (1990). The semiotic subject of cultural psychology. In L. Pervin (Ed.), Handbook of personality: Theory and research (pp. 399-416). New York: Guilford Press.

Sih, A., \& Bell, A. M. (2008). Insights from behavioral syndromes for behavioral ecology. Advances in the Study of Behavior, 38, 277-281.

Simon, H. A. (1993). Homo rationalis: die Vernunft im menschlichen Leben. Frankfurt am Main: Campus. [engl. Simon, H. A. (1983). Reason in human affairs. Stanford, California: University Press.]

Sinn, D. L., \& Moltschaniwskyj, N. A. (2005). Personality traits in dumpling squid (Euprymna tasmanica): context-specific traits and their correlation with biological characteristics. Journal of Comparative Psychology, 119, 99-110.

Soldz, S., \& Vaillant, G. E. (1999). The big five personality traits and the life course: a 45-year longitudinal study. Journal of Research in Personality, 33, 208-232.

Spearman, C. (1910). Correlation calculated from faulty data. British Journal of Psychology, 3, 271-295.

Sprung, L., \& Sprung, H. (1984). Grundlagen der methodologie und methodik der psychologie. Berlin: Deutscher Verlag der Wissenschaften. 
Standford, C. B. (1998). The social behavior of chimpanzees and bonobos: empirical evidnce and shifting assumptions. Current Anthropology, 39, 399-420.

Stern, W. (1935). Allgemeine Psychologie auf personalistischer Grundlage. Haag: Nijhoff. English translation (1938). General Psychology From the Personalistic Standpoint. New York: Macmillan.

Suomi, S. J. (2005). How gene-environment interactions shape the development ofimpulsive aggression in rhesus monkeys. In D. M. Stoff \& E. J. Sussman (Eds.), Developmental psychobiology of aggression (pp. 252-268). New York: Cambridge University Press.

Tellegen, A. (1993). Folk concepts and psychological concepts of personality and personality disorders. Psychological Inquiry, 4, 122-130.

Thelen, E., \& Smith, L. B. (1993). A dynamic systems approach to the development of cognition and action. Cambridge: MIT Press.

Thurstone, L. L. (1934). The vectors of mind. Psychological Review, 41, 1-32.

Tinbergen, N. (1963). On aims and methods of ethology. Zeitschrift für Tierpsychologie, 20, 410-433.

Tolman, E. (1948). Cognitive maps in rats and men. Psychological Review, 55, 189-208.

Toomela, A. (2008). Variables in psychology: a critique of quantitative psychology. Integrative Psychological and Behavioral Science, 42, 245-265.

Toomela, A. (2009). How methodology became a toolbox-and how it escapes from that box. In J. Valsiner, P. Molenaar, M. Lyra, \& N. Chaudhary (Eds.), Dynamic process methodology in the social and developmental sciences (pp. 45-66). New York: Springer.

Toomela, A. (2010a). In A. Toomela \& J. Valsiner (Eds.), Methodological thinking in psychology: 60 years gone astray? (pp. 1-26). Charlotte: Information Age Publishers.

Toomela, A. (2010b). In S. Salvatore, J. Valsiner, A. Gennaro, \& J. B. Simon Travers (Eds.), Yearbook of idiographic science (Vol. 2). Rome: Firera \& Liuzzo Group.

Toomela, A. (2011). Travel into a fairy land: a critique of modern qualitative and mixed methods psychologies. Integrative Psychological and Behavioral Science, 45, 21-47.

Toomela, A., \& Valsiner, J. (Eds.). (2010). Methodological thinking in psychology: 60 years gone astray? Charlotte: Information Age Publishers.

Trendler, G. (2009). Measurement theory, psychology and the revolution that cannot happen. Theory and Psychology, 19, 579-599.

Trut, L. N. (1999). Early canid domestication: the farm-fox experiment. American Scientist, 87, 160-169.

Uher, J. (2008a). Three methodological core issues of comparative personality research. European Journal of Personality, 22, 475-496.

Uher, J. (2008b). Comparative personality research: methodological approaches. European Journal of Personality, 22, 427-455.

Uher, J. (2009). Botanical personality? On the origins of consistent individual differences in behavior. In J. Uher Comparative research on personality in Animalia and Plantae. PhD-Dissertation. Berlin: Freie Universität.

Uher, J. (2011a). Individual behavioral phenotypes: an integrative meta-theoretical framework. Why 'behavioral syndromes' are not analogues of 'personality'. Developmental Psychobiology, 53, 521548.

Uher, J. (2011b). Personality in nonhuman primates: What can we learn from human personality psychology? In A. Weiss, J. E. King, \& L. Murray (Eds.), Personality and behavioral syndromes in nonhuman primates. New York: Springer.

Uher, J., \& Asendorpf, J. B. (2008). Personality assessment in the great apes: comparing ecologically valid behavior measures, behavior ratings, and adjective ratings. Journal of Research in Personality, 42, $821-838$.

Uher, J., Asendorpf, J. B., \& Call, J. (2008). Personality in the behaviour of great apes: temporal stability, cross-situational consistency and coherence in response. Animal Behaviour, 75, 99-112.

Utz, K. (2005). Philosophie des Zufalls: Ein Entwurf. Paderborn: Schöningh.

Valsiner, J. (1998). The guided mind. Cambridge: Harvard University Press.

Valsiner, J. (2009). Cultural psychology today: innovations and oversights. Culture \& Psychology, 15, 539.

Valsiner, J. (2012). A guided science: History of psychology in the mirror of its making. New Brunswick: Transaction Publishers.

Van Geert, P., \& van Dijk, M. (2002). Focus on variability: new tools to study intra-individual variability in developmental data. Infant Behavior \& Development, 25, 340-374.

Veltkamp, G. M., Recio, G., Jacobs, A. M., \& Conrad, M. (2012). Is personality modulated by language? Evidence from bilinguals' NEO-FFI scores. International Journal of Bilingualism. Published online before print April 16, 2012, doi:10.1177/1367006912438894 
Voelklein, C., \& Howarth, C. (2005). A review of controversies about social representations theory: a British debate. Culture \& Psychology, 11, 431-454.

Von Mises, R. (1928/1981). Probability, statistics and truth. New York: Dover Publications.

Vygotsky, L. S. (1962). Thought and language. Cambridge: MIT Press.

Wagner, W., Farr, R., Jovchelovitch, S., Lorenzi-Cioldi, F., Marková, I., Duveen, G., et al. (1999). Theory and method of social representations. London: LSE Research Online.

Wagoner, B. (2009). The experimental methodology of constructive microgenesis. In J. Valsiner, P. Molenaar, N. Chaudhary, \& M. Lyra (Eds.), Handbook of dynamic process methodology in the social and developmental sciences (pp. 99-121). New York: Springer.

Weber, M. (1949). In E. Shils \& H. Finch (Eds.), The methodology of the social sciences. New York: Free Press.

Webster's new international dictionary (1925). (2nd unabridged ed.) Springfield: Merriam.

Weinstein, T. A. R., \& Capitanio, J. P. (2008). Individual differences in infant temperament predict social relationships of yearling rhesus monkeys (Macaca mulatta). Animal Behaviour, 76, 455-465.

Weinstein, T. A. R., \& Capitanio, J. P. (2012). Longitudinal stability of friendships in rhesus monkeys (Macaca mulatta): individual- and relationship-level effects. Journal of Comparative Psychology, 126, 97-108.

Westen, D. (1996). A model and a method for uncovering the nomothetic from the idiographic: an alternative to the five-factor model. Journal of Research in Personality, 30, 400-413.

Westen, D. (1999). The scientific status of unconscious processes: is Freud really dead? Journal of the American Psychoanalytic Association, 47, 1061-1106.

Whitehead, A. N. (1929). Process and reality. New York: Harper.

Whorf, B. L. (1958). Language and stereotypes. In E. E. Maccoby, T. M. Newcomb, \& E. L. Hartley (Eds.), Readings in social psychology (3rd ed., pp. 1-9). New York: Holt.

Wiggins, J. S. (1979). A psychological taxonomy of trait-descriptive terms: the inter-personal domain. Journal of Personality and Social Psychology, 37, 395-412.

Wiggins, J. S. (2003). Paradigms of personality assessment. New York: Guilford.

Witkowski, S. R., \& Brown, C. H. (1978). Lexical universals. Annual Review of Anthropology, 7(427), 451.

Wright, J. C., \& Mischel, W. (1987). A conditional approach to dispositional constructs: the local predictability of social behavior. Journal of Personality and Social Psychology, 55, 454-469.

Wright, J. C., \& Zakriski, A. L. (2003). When syndromal similarity obscures functional dissimilarity: distinctive evoked environments of externalizing and mixed syndrome children. Journal of Consulting and Clinical Psychology, 71, 516-527.

Wundt, W. M. (1904). Principles of physiological psychology. London: Allen.

Jana Uher received her PhD from Freie Universität Berlin in 2009 where she is heading the research group Comparative Differential and Personality Psychology that she founded in 2010. Her research is transdisciplinary concentrating on philosophy-of-science issues of psychological and behavioural research on individuals in humans and nonhuman species from species-comprehensive and culture- and speciescomparative perspectives. For demonstrating her metatheoretical and methodological developments, she has been working empirically with primate species in particular human children, the great apes, capuchin monkeys, and various macaques, and with plant species using noninvasive experimental and observational studies. Additionally, she has been conducting assessment studies with adult observers of young children and with human observers of nonhuman individuals respectively. Her empirical research projects are rooted in international and national collaborations with scholars from different disciplines. She has been working at the Max Planck Institute for Evolutionary Anthropology in Leipzig (2003-2005), and has also been a visiting scholar at the Institute of Cognitive Sciences and Technologies, National Research Council of Italy (ISTC-CNR) in Rome (2011-2012). 